\title{
EL LEGADO MUSICAL DE PEDRO DE ARDANAZ Y MIGUEL DE AMBIELA. DOS INVENTARIOS CON PAPELES DE MÚSICA EN LATÍN DE LA CATEDRAL DE TOLEDO (1713 Y 1737)
}

\author{
THE MUSICAL LEGACY OF PEDRO DE ARDANAZ AND \\ MIGUEL DE AMBIELA. TWO INVENTORIES WITH PAPERS \\ OF MUSIC IN LATIN FROM THE CATHEDRAL OF TOLEDO (1713 AND 1737)
}

\author{
Carlos Martínez Gil \\ Archivo de Música de la catedral de Toledo \\ cmargil@telefonica.net \\ ORCID iD: https://orcid.org/0000-0002-0802-3181
}

\begin{abstract}
Resumen
Con la perspectiva de tener ya casi terminado el Catálogo de fondos musicales conservados de la catedral de Toledo (16001900), el autor del artículo nos remite a dos inventarios que recogen una ingente lista de obras de música en latín, la mayoría de ellas desaparecidas. Las fechas de los inventarios, 1713 y 1737, reflejan la actividad musical de dos maestros de dilatada trayectoria al frente de la capilla toledana: Pedro de Ardanaz (1674-1706) y Miguel de Ambiela (1710-1733). Por un lado, el inventario redactado tras la muerte de Ardanaz nos describe las características del repertorio latino practicado en los templos españoles durante el siglo XVII; por otro, el documento relacionado con Ambiela supone una lanzadera de las propuestas musicales que, en el mismo ámbito, se van a ir desarrollando a lo largo del siglo XVIII. Los inventarios de música son un complemento necesario para la elaboración de los catálogos de obras conservadas, ya que éstos solo nos dan una información incompleta sobre la historia musical de una institución. Para ello, un catálogo de música siempre debería ir acompañado de la sucesión lineal de los inventarios hallados, de tal modo que nos permitan valorar la actividad musical de un determinado lugar a través del repertorio que hay (catálogo), pero también del que hubo (inventarios).
\end{abstract}

\section{Palabras clave \\ Inventarios, archivo, catálogo, catedral de Toledo, maestros de capilla, obras latinas, papeles de música, Ardanaz, Ambiela.}

\begin{abstract}
With a view to finishing already almost all the Catalogue of musical collection preserved of the cathedral of Toledo (16001900), the author of the article refers to two inventories that gather an enormous list of works of music in Latin, most of them disappeared. The dates of the inventories, 1713 and 1737, reflect the musical activity of two masters with a large trajectory at the head of the chapel of Toledo: Pedro de Ardanaz (1674-1706) and Miguel de Ambiela (1710-1733). On the one hand, the inventory written after Ardanaz's death describes the characteristics of the Latin repertory practiced in the Spanish temples during the $18^{\text {th }}$ century; on the other hand, the document related to Ambiela means an impetus for the musical proposals that, in the same field, would going to be developed throughout the 18th century. These musical inventories are a complement necessary to elaborate the catalogues of preserved works, since these only give us an incomplete information about the musical history of an institution. To that end, a catalogue of music always should be accompanied of the linear succession of the found inventories, in such a way that they allow us to value the musical activity of a certain place through the repertory that currently exists (catalogue) but also of the one that once existed (inventories).
\end{abstract}

\section{Key words}

Inventories, archive, library catalogue, cathedral of Toledo, chapel master, latin works, music papers, Ardanaz, Ambiela. 
Era común que, cuando un maestro de capilla tomaba posesión de su puesto en una catedral, el Cabildo encargase la elaboración de un inventario de toda la música que hubiera estado bajo la responsabilidad del maestro anterior. Además, los mismos maestros eran propietarios de sus obras, a las que podían añadir las de otros autores que habían ido coleccionando a lo largo de su carrera profesional, de manera que, cuando aquellos morían, sus herederos solían ofrecer el patrimonio musical del difunto a cambio de una gratificación económica. Por lo general, el Cabildo aceptaba la oferta, pasando esta lista de obras a formar parte de los fondos musicales de la catedral, quedando estos a cargo del nuevo maestro de capilla.

En los últimos años, hemos ido sacando a la luz en el Archivo catedralicio de Toledo una buena parte de este tipo de documentación -traspapelada en legajos misceláneos-, que nos va dando a conocer nuevos detalles sobre el repertorio que se practicaba en el culto de la catedral en épocas pasadas, ya que, lamentablemente, la mayoría de la música que se cita en ella, ha desaparecido. En cualquier caso, la secuenciación de varios inventarios encontrados nos permite hacer un seguimiento tanto de los fondos musicales conservados, como de aquellos que ya no existen; en lo que se refiere a estos últimos, la falta de uso pudo ser la causa de la desaparición de estas obras antiguas en sucesivos inventarios, que dejan de mencionarlas. La documentación suele citar las adquisiciones, pero no las ausencias. En 1710, en las gestiones del Cabildo para la adquisición de los papeles de música que pertenecieron a Pedro de Ardanaz, se advierte al maestro vigente, Miguel de Ambiela, que tuviera a buen recaudo toda la música que se confiaba a su custodia, y que la guardase

“con los demás que [h]ay en esta S[an]ta Iglesia en parte donde no se pierdan, como parece [h]a sucedido con los más de los que havía por el poco resguardo del cajón que está en las tribunillas"1.

Estos inventarios representan un complemento de especial interés para la elaboración del Catálogo de obras musicales conservadas actualmente en el Archivo de la Catedral de Toledo (que estamos a punto de terminar) ${ }^{2}$, y sobre todo contextualizan el repertorio que se utilizó allí a lo largo del siglo XVIII,

1 Actas Capitulares de la Catedral de Toledo, 51, 28.02.1710, ff.218v.-219r. (ACT).

2 Tras varios años de trabajo (con desigual dedicación, ya que es un proyecto realizado por iniciativa personal), estoy cerca de terminar el Catálogo junto con mi colaborador Miguel Ángel Ríos Muñoz. El Catálogo recoge los fondos musicales conservados pertenecientes al período comprendido entre los años 1600 y 1900, ya que la catalogación correspondiente a los libros de coro polifónicos debe correr a cargo de Michael Noone, de quien esperamos nos ofrezca lo más pronto posible sus expertas conclusiones. Nuestro Catálogo va a ser publicado en 2019 en la espléndida colección "Primatialis Ecclesiae Toletanae Memoria", que edita el Cabildo de la Catedral de Toledo, bajo la dirección de Ángel Fernández Collado. teniendo en cuenta que, de las cerca de $1.200^{3}$ obras que hemos recogido hay una mayoría de ellas, con un $85 \%$, pertenecientes a esta centuria. En nuestro Catálogo llamará la atención la escasa presencia de obras pertenecientes al siglo XVII que se han conservado (poco menos de treinta ${ }^{4}$; todas latinas), y más si reparamos en el rico patrimonio de las mismas que hubo hasta mediados del siglo XVIII, si nos atenemos a las referencias de los dos inventarios que van a ser objeto de este estudio (1713 y 1737). Las posibles razones de su desaparición ya las he expuesto hace un momento: la falta de interés por conservar una música que, en su mayor parte, había caído en desuso. Estos primeros inventarios dieciochescos solo recogen repertorio de obras en latín, lo que nos indica que éste era el que podría ser reutilizado más allá del preciso momento de su creación, y de ahí su mayor perdurabilidad en el tiempo. Los villancicos en castellano, cuya primera muestra conservada en el Archivo data de $1735^{5}$, tenían un valor más efímero, pues eran concebidos bajo la intención de ser utilizados para una sola ocasión, y de ahí el poco esmero que se tuvo para su conservación.

En comparación con la escasez de inventarios encontrados en el siglo XVII, su abundancia desde comienzos del XVIII en adelante ${ }^{6}$, nos describe la intrahistoria de los fondos musicales de la catedral de Toledo, permitiéndonos saber así qué permanece, qué se pierde y qué se añade a lo largo de más o menos dos centurias. Ante la necesidad de completar el mapa de los fondos musicales conservados en los principales centros religiosos de la Península, labor que poco a poco se va cumpliendo, resultaría muy interesante que fuéramos dando a conocer los "otros catálogos", esto es, los de los inventarios que citan las obras que se fueron utilizando con el paso del tiempo y que no han llegado hasta la actualidad, reunidos en cada lugar en formato de publicaciones unificadas. De este modo, no solamente podríamos valorar el papel jugado por la música en una determinada catedral, colegiata o iglesia a través de lo que se ha conservado, sino también de lo que hubo y sonó a lo largo de su trayectoria y que se perdió por el camino.

Sería deseable, pues, que me planteara hacer un estudio de la música en la catedral de Toledo en el siglo XVIII a la luz de los inventarios conservados, y a buen seguro ampliaría con ello el conocimiento de aquella completando la información de otras fuentes documentales más habituales. Pero de momento, y debido a la limitación de espacio, voy a seleccionar solamente dos inventarios que, como podremos comprobar, resultan de un elevado interés para percibir los cambios estilísticos que se operan en los templos españoles con el paso del siglo XVII al XVIII.

3 Hasta que no demos por cerrado el Catálogo definitivamente esta cifra no estará precisada.

424 de autor conocido y puede que media docena anónimas.

5 Se trata del villancico $5^{\circ}$ para los maitines de Navidad de 1735 de Jaime Casellas, que comienza cantando "¿No es buen tema el de aquel Niño...?", a 10 voces en 3 coros, 2 violines y acompañamiento.

6 Conservamos inventarios fechados en 1706, 1737, 1775, 1780, $1793,1795,1803,1807,1819,1825,1832$, así como otros no fechados que podrían estar escritos hacia 1789, 1820 y 1900. 
Los dos inventarios que he elegido para analizar en este trabajo están separados entre sí por poco más de 20 años, fechados respectivamente en 1713 y 1737 , y ambos representan, por sus contenidos, dos maneras diferentes de concebir la música en los templos españoles ${ }^{7}$. Por un lado, el primero recoge obras que obedecen a la tradición coral proveniente del siglo anterior, mientras que el segundo incluye ya repertorio que denota una incipiente evolución en la utilización de las voces de una manera más flexible y variada, así como la inclusión de instrumentos, como violines y oboes, que progresivamente van cobrando mayor protagonismo.

El inventario fechado en 1713 corresponde a las obras latinas que pertenecieron a Pedro de Ardanaz, un músico especialmente ligado a la catedral de Toledo, ya que entró en ella como seise a la edad de 8 años en 1647, procedente de Tafalla, en Navarra, y posteriormente ostentó el cargo de maestro de capilla desde 1674 hasta su muerte en 1706. Ardanaz se formó en Toledo con el maestro Tomás Micieces, y allí coincidió como seise con compañeros como Miguel de Irízar y Matías Durango $^{8}$. De sus 68 años de vida, pasó 44 en Toledo, exceptuando sus 8 años de infancia y los 16 como maestro de capilla en la catedral de Pamplona entre 1658 y 1674. Es el maestro que más tiempo estuvo al frente de la capilla de Toledo en todo el siglo XVII (32 años), siendo uno de los principales representantes de la música española durante el período regentado por el último monarca de la casa de los Austrias, Carlos II, junto a nombres tan señalados como los de Sebastián Durón, Cristóbal Galán, Juan Hidalgo -estos relacionados con la Corte-, José de Vaquedano, Juan Bonet de Paredes, Matías Veana o el mencionado Miguel de Irízar, por citar solo algunos.

El inventario de 1737 recoge el riquísimo patrimonio de obras latinas que pertenecieron a Miguel de Ambiela, maestro en Toledo entre $1710^{9}$ y 1733 , etapa final de una inquieta trayectoria profesional que le llevó sucesivamente por Daroca, Lleida, Jaca, El Pilar de Zaragoza y las Descalzas Reales de Madrid. A Ambiela se le puede considerar un músico-puente que une la tradición coral del repertorio practicado en las catedrales españolas en el siglo anterior, con las innovaciones que se van incorporando en las primeras décadas de la monarquía borbónica, y que poco a poco van a ir configurando un nuevo estilo bien

7 El presente artículo es una primera parte que pide continuación con el estudio de los inventarios posteriores que hasta el momento hemos encontrado. Pero dado que los plazos de publicación de las revistas de investigación musical son largos, lentos e inciertos, procuraré abordar el asunto en el prólogo de la próxima edición del Catálogo de fondos musicales del Archivo Capitular de Toledo (1600-1900), ya que el contenido de estos inventarios subsiguientes tienen más que ver con la música que se ha conservado.

8 Irízar fue posteriormente maestro en Segovia y Durango trabajó en distintos lugares como Logroño, Palencia, Falces y Santo Domingo de la Calzada.

9 Nótese que, entre la muerte de Ardanaz y la toma de posesión de Ambiela, median cuatros años (1706-1710) que separan el final del magisterio de uno con el comienzo del otro. En este breve período de tiempo, Juan Bonet de Paredes gastó sus últimos años de profesión. diferenciado del de las etapas anteriores. El amplio contenido de este inventario, como veremos, contiene una miscelánea de obras que reconocen la vigencia de maestros clásicos del pasado conviviendo con los nuevos representantes de la música religiosa española del momento.

El paso de Miguel de Ambiela por la catedral de Toledo supuso la incorporación de una serie de novedades, que sirvieron para que la música que en ella se hacía fuera adaptándose paulatinamente al estilo que se iba imponiendo en los templos españoles desde comienzos de la centuria. La proximidad de la Corte, de donde procedía Ambiela -venía de trabajar en las Descalzas Reales-, obligaba aún más a que una institución tan señera como la de Toledo no se quedara atrás en el abrillantamiento de la música que adornaba sus ceremonias. Algunas de estas innovaciones que se fueron añadiendo a lo largo del magisterio de Ambiela repercutieron directamente en la evolución del repertorio que allí se mostraba; entre las más destacadas, podemos señalar el asentamiento en plantilla de los violines y oboes en 1717, el reforzamiento del acompañamiento continuo con la inserción del contrabajo de violón (1728) y de una pareja de violones, a los que se les exigía además labores concertantes; o la necesidad de contar con tres organistas para cubrir todas las funciones con garantías. Entre estos últimos, hay que subrayar el llamativo liderazgo que ejerció Joaquín Martínez de la Roca, desde su acceso a la capilla en 1722, quien compartirá responsabilidades con Ambiela en los últimos años del magisterio de éste, así como en los primeros años de su sucesor, Jaime Casellas.

A pesar del poco margen de años que media entre los dos inventarios, bien pareciera que se tratase de documentos que proceden de centros religiosos diferentes, pues el de 1737 nombra ya pocas obras de las que se citaban en el de 1713. De las 196 posibles obras de Ardanaz que se recogían en el primer documento tan solo quedan 7 en la lista de Ambiela, sin la total certeza de que el salmo Laudate, las 5 Salves y el Miserere a 12 sean las mismas que se mencionan 24 años antes. ¿Qué se hizo entonces con tantas obras del maestro Ardanaz? ¿Realmente ya estaban perdidas o no fueron consideradas para formar parte de la relación de composiciones legadas por Ambiela? Lo cierto es que en la actualidad tan solo se conservan 6 obras de Pedro de Ardanaz en el Archivo toledano ${ }^{10}$, de las que ninguna forma parte del inventario de 1737; tan solo podemos pensar que los cuatro salmos conservados hoy podrían formar parte de la larga lista de obras del maestro navarro del primer inventario.

En el caso de Ambiela sucede algo parecido: se conservan 21 obras $^{11}$, de las cuales ocho son salmos de vísperas y que muy

10 Missa a 8 para Nuestra Señora del Rosario (solo el acompanamiento continuo), Motete In illo tempore a 4, Beatus vir a 10, y tres Letatus sum, a 13, 12 y 10 voces.

11 Exceptuando los libros de coro que contienen obras compuestas en el estilo polifónico clásico. Los libros de coro 23, 24 y 25 incluyen obras de Ambiela junto a las de otros maestros, pero el n ${ }^{\circ} 2$ está dedicado plenamente a él, con dos antífonas y 8 misas con sus respectivos motetes. Las obras conservadas son las siguientes: Salve Regina a 8 (fragmento), Te Deum a 6, Invitatorio a la Expectación y Descensión 
probablemente formen parte de la lista de los que se citan en el inventario de 1737, así como de los tres mencionados en el de Ardanaz. Una obra que se repite en ambos inventarios es el $O f$ cio de Nuestra Señora de los Dolores, compuesta de vísperas, misa y motete, conservado hoy de manera fragmentada, y que se confeccionó de manera cuidadosa "en cuadernos de pergamino metidos en su caja de madera". Por otro lado, el hecho de que a la muerte de Ardanaz figurasen 9 obras de Ambiela entre el conjunto de papeles de música que estaban a su cargo, invita a pensar que éste estuviera ya en el punto de mira para ser acreedor al puesto que ocuparía a partir de 1710. Poco queda pues de las 111 obras del compositor aragonés, que Martín Arevalillo, su albacea y heredero, entregó al cabildo en 1737.

Si nos detenemos ahora en comprobar qué nos ha llegado de los otros maestros mencionados en ambos inventarios, repararemos en que asimismo la mayoría de las composiciones han desaparecido. En el documento relacionado con Ardanaz se citan tan solo 8 nombres, mientras que en el de Ambiela -más rico en detalles- son 27. Pues bien, del inventario de 1713 tan solo puedo reconocer dos magnificats de Micieces en el catálogo actual, mientras que en el de 1737 estas obras no aparecen mencionadas y sí otras nueve que actualmente se conservan ${ }^{12}$. De todas formas, entre las aproximadamente 1.200 composiciones recogidas en el catálogo que estamos terminando, tan solo 66 son anteriores al comienzo del magisterio de Jaime Casellas en 1734, sucesor de Miguel de Ambiela ${ }^{13}$.

\section{EL INVENTARIO DE OBRAS EN LATÍN QUE FUERON DE PEDRO DE ARDANAZ}

Tras la muerte del maestro Pedro de Ardanaz el 11.10.1706, dos días después, su hermana Sebastiana ofrecía al cabildo "todos los papeles que el dicho su hermano tenía tocantes a su ministerio" 14 . La oferta, sin que se mencionase nada sobre compensación económica alguna, se acogió de buen grado. Sin embargo, en febrero de 1710 aún no se había producido la entrega de dichos papeles de música, y en esta ocasión ya no se mencionaba como negociadora a la hermana del difunto Ardanaz, sino al marido de ésta, el ministril sacabuche de la catedral Juan de Medina, quien se mostraba dispuesto a cumplir el trato ${ }^{15}$. Dos años después, en octubre de 1712, el asunto seguía sin resol-

de Nuestra Señora a 7, Invitatorio e Himno de Navidad, Nunc dimittis a 10, fragmentos de una misa, Oficio de Nuestra Señora de los Dolores (incompleto), 8 salmos de vísperas, In exitu Israel a 8 y un villancico a Santa Rita.

12 Se trata de tres Magnificats de Sebastián Alfonso, Diego Cáseda y Francisco Valls, un Credidi de Matías Veana y otro de Carlos Patiño, un Laetatus de este último, un Beatus vir de José de Torres, Memento Domine de Bonet de Paredes y los fragmentos de la Missa a 7 de Sebastián Durón. de coro.

13 Sigo sin contar con las que se integran en los grandes libros

14 ACT, 49, 13.10.1706, ff. 317v.-318r.

15 ACT, 51, 28.02.1710, ff.218v.-219r. verse, y esta vez el Cabildo sí mostraba su malestar expresando "el reparo de que la parte del heredero parece que no ha andado tan bien como debiera, supuesto que ha querido dar a entender que no expresó el Maestro esta oferta, y se está sin entregarlos"16. De todo esto se deduce la clara intención del ministril de sacar un beneficio económico que no llega a satisfacer según sus previsiones. En ese momento, el cabildo manifiesta su verdadero interés por los papeles de música de Ardanaz que reclama, expresando que éstos "son muy primorosos porque el dicho Maestro lo fue en la composición de todo lo que toca a Latín", y es entonces cuando se empieza a hablar de una gratificación económica para terminar con el asunto, resolviéndose entonces recibirlos, pero "que sea por inventario que haya de quedar en la Contaduría de la Obra, y que se pongan en las Tribunillas del Coro" ${ }^{17}$. Finalmente, en abril de 1713, casi siete años después de iniciarse el proceso, los papeles de música que dejara el maestro Ardanaz quedaban a cargo del maestro Ambiela, tras haberse librado una remuneración de 20 doblones de a dos escudos de oro a Juan de Medina y a su esposa Sebastiana de Ardanaz ${ }^{18}$.

De todo este inexplicable y largo litigio creo intuir una mala relación entre el maestro de capilla vigente, el recién llegado Miguel de Ambiela, y el ministril sacabuche Juan de Medina. Tres años después de este prolongado incidente, se vuelve a mencionar en las actas a este ministril con motivo de la introducción de los violines en la plantilla de la capilla de Toledo. Como defensa para la aceptación con carácter estable de este nuevo instrumento, se argumentaba que

“...están tan introducidos en muchas capillas, y pueden suplir en parte la falta que hay hoy de voces, además de que regularmente se han tenido los instrumentos que más se estilan, y se han dejado otros como es el Archilaúd, que ya no hay días ha; y es mucho lo que hay de esotros de caña en que entra el sacabuche que hace poco o nada, ni debía repararse en que los violines se usen en los teatros, pues lo mismo sucede con el Arpa y Violón, y sin embargo los hay en el Choro..." 19

Muy bien se podría considerar esta cita como un dardo expresamente dirigido al ministril Juan de Medina como represalia del maestro de capilla a la desagradable disputa por los papeles de Ardanaz; pero también, y de manera indirecta, estas apreciaciones podrían interpretarse como una crítica a la música de éste por parte de Ambiela por considerarla ya caduca ante el envite del nuevo estilo y con el que se identifica plenamente al defender con denuedo la incorporación de violines y oboes. ¿Será esta la causa de que en el inventario de 1737 apenas quede rastro de la abundante obra de Ardanaz que figura en el documento de 1713 ?

El inventario que se redactó en 1713 contiene un total de 223 referencias mayoritariamente de obras del que fuera su

16 ACT, 52, 21.10.1712, ff.288r.-288v.

17 Ibid.

18 ACT, 53, 06.04.1713, ff.62r.-62v.

19 ACT, 54, 17.12.1716, ff.365v.-366v. 
poseedor, Pedro de Ardanaz. Además, el documento tiene un anexo en el que se añaden otras 24 obras de Juan de Padilla, que fueron adquiridas mediante una donación procedente de la catedral de Córdoba, en la que su heredero cumplía el deseo de este compositor para depositarlas en Toledo tras su fallecimiento ${ }^{20}$. Juan de Padilla fue, precisamente, el maestro de capilla que en Toledo precedió a Pedro de Ardanaz, y desempeñó el cargo entre 1662 y 1673 .

Por lo tanto, si descontamos el añadido con obras de Padilla, ya que no forma parte del documento matriz, podemos hablar de que en él se citan nada menos que 195 obras de Ardanaz y 28 de otros compositores. Doy por supuesto que aquellas obras sin indicación de autor están compuestas por el maestro navarro, pues en las pocas ocasiones en las que esto sucede se adjunta con toda intención el nombre del autor del ítem mencionado. Solo aparecen escritos nueve nombres de músicos españoles diferentes al de Ardanaz, dispersos a lo largo del manuscrito, ya que el criterio general que organiza el documento se rige por la agrupación de géneros litúrgicos, de acuerdo con el siguiente orden: misas (10), motetes (28), secuencias (2), nonas (4), salmos de vísperas (37), magnificats (12), himnos de vísperas (15), salmos de completas (13), letanías a la Virgen y al Santísimo Sacramento (8), salves (14), salmos de maitines (2), oficio de difuntos e invitatorios (7) y, finalmente, repertorio para la Cuaresma: motetes (22), lamentaciones distinguiendo si son para el miércoles, jueves o viernes santo (8), y misereres (26). Después de las letanías hay un paréntesis en el documento en el que se hace recuento numérico de "los Papeles de Latín del Maestro Don Pedro de Ardanaz", tras el cual se cita un pequeño bloque de obras de otros autores, como Solana, Ambiela, Padilla, García y Rogier.

De estos nueve compositores citados, cuatro no están relacionados con la vida musical de Toledo, y se les podría considerar como maestros ya lejanos en el tiempo, puesto que estuvieron activos sobre todo en la primera mitad del siglo XVII (Carlos Patiño, Juan Pérez Roldán y Gerónimo Vicente), a excepción de Philippe Rogier, uno de los principales representantes de la capilla flamenca de Felipe II, y protagonista del libro de coro $\mathrm{n}^{\circ} 15$ conservado en el archivo ${ }^{21}$. Los otros cinco sí estaban relacionados con la capilla de Toledo: tres de ellos han sido maestros en épocas pasadas, que son Vicente García,

20 "Vista una carta que en 17 de Junio escribe al M[aest]ro de Capilla de esta S[an]ta Ig[lesi]a, D. Bernardo de Medina y Corpas que parece lo es de la Iglesia de Cordova, en que refiriendo haver muerto su M[aest]ro D. Juan Pacheco en cuyo poder paraban ciertos papeles de Missas y Psalmos que dejo p[ar]a esta S[an]ta Ig[lesi]a el M[aest] ro D. Juan de Padilla, y que cumpliendo con la disposición de ambos Maestros lo remitirá si el Cab[ild]o fuese servido admitirlos: acordaron que D. Pedro Ardanaz responda que los imbie, ya que no lo hiço el d[ic] ho D. Juan Pacheco con la puntualidad que debía por el deseo que se dice tuvo de copiarlos". ACT, 49, 01.07.1706, f.264r.

21 Se trata del volumen publicado en Madrid en 1598 que contiene cinco misas de Rogier y una de Gery de Ghersem, la primera de ellas, la célebre Missa Philippus II Rex Hispaniae, a 4 voces, y que se conserva en varios archivos españoles.
Tomás Micieces y Juan de Padilla, y uno, Miguel de Ambiela, lo será ${ }^{22}$. El nombre que resta, Matías Solana, representado con unas vísperas y una misa, fue organista $2^{\circ}$ desde 1689 hasta la fecha de su muerte en 1722, al amparo de su tío, José Solana, organista principal.

El esquema que se repite en cada cita del inventario como base para la identificación y distinción de cada obra recoge los siguientes datos: género litúrgico, número de voces, dedicación de la fiesta (en el caso de que sea para un día determinado), y el tono. La especificación de los tonos en la mayoría de las obras podría permitir un estudio sobre cuáles son los más usados en los distintos géneros de música en latín. Una primera estadística nos revela que los preferidos son los tonos segundo y octavo. Este dato, como rasgo inherente de la obra citada, se referirá cada vez menos en los inventarios a medida que avanzamos en el siglo XVIII.

En escasas ocasiones se mencionan instrumentos musicales y, cuando sucede, son las chirimías las que complementan a las voces representando a uno de los coros. Llama la atención la relación instrumental que conlleva el himno Monstra te esse que, por su carácter procesional ${ }^{23}$, implica una participación obligada de los ministriles ${ }^{24}$. Ninguna de las obras de Ardanaz lleva violines, lo cual era demasiado prematuro para Toledo, si bien en la vecina corte Sebastián Durón ya los estaba usando con asiduidad; tan solo se nombran en el documento en las obras de Ambiela, y en una de ellas, acompañados por oboes (Missa a ocho con violines y oboes).

Otro campo de análisis de gran interés es el del número de voces empleado, y que está detallado en la mención de todas las obras del inventario. Está claro que predominan las composiciones a 8 y 12 voces, en dos y tres coros respectivamente, pero podemos encontrarnos con una gran variedad de formaciones que oscilan entre las 4 y las 20 voces; dos de las misas citadas emplean un gran número de voces: la Missa sobre el himno Quem terra, de Miguel de Ambiela, y la Missa de a veinte vozes con chirimías de quinto tono de Pedro de Ardanaz. Mientras la mayoría de géneros litúrgicos juegan con distintas combinaciones en el número de voces, hay otros que se caracterizan por presentarse siempre con una formación fija; es el caso de los motetes para las dominicas y ferias de quaresma, que todos son a 4 voces.

Un caso especial son los misereres para la Semana Santa, de los que se computan al final del inventario un total de 21 , todos al parecer de Ardanaz. De ellos, seis especifican el número de voces, oscilando entre seis y doce, pero al resto se los califica

22 Lo será en el momento de la muerte de Ardanaz y lo es en el de la redacción del inventario.

23 Es un verso del himno Ave maris stella, que se canta en las estaciones durante las procesiones de la Virgen.

24 En el inventario se citan cuatro:

$\checkmark$ Un Monstra te esse Matrem, a quatro con instrumentos.

$\checkmark$ Otro a quatro con instrumentos.

$\checkmark$ Otro a tres con instrumentos.

$\checkmark$ Otro a tres con dos vajones del Maestro Micieces. 
como Misereres de los grandes, lo que nos hace pensar en una mayor participación vocal, del mismo modo que se desarrollarán en el siglo XVIII cuando alguno de los misereres de Jaime Casellas llegarán a contar hasta con 18 voces distribuidas en 5 coros que se colocaban en diferentes tribunillas alrededor del crucero de la catedral.

Por último, algunas de las misas mencionadas informan del material musical sobre el que están basadas, la mayor parte en motetes e himnos religiosos de la manera habitual ${ }^{25}$. Pero hay dos que se desmarcan de este recurso y que aportan singularidad en este género al tratarse de dos misas parodia no basadas en composiciones latinas: por un lado, la Missa de Batalla de Pérez Roldán, una variedad muy demandada en la España del siglo XVII, y por otro, la Missa sobre Entre Fragosas Breñas, de Vicente García, cuyo texto es un soneto dedicado a San Jerónimo que viene circulando por la Península desde el siglo $\mathrm{XVI}^{26}, \mathrm{y}$ del que su autor quizá hubiera hecho alguna composición en romance previamente a la Missa.

\section{EL INVENTARIO DE OBRAS EN LATÍN QUE FUERON DE MIGUEL DE AMBIELA}

Cuatro años después de la muerte de Miguel de Ambiela, el 29.03.1733, su heredero y albacea ofrece al cabildo el patrimonio musical del maestro fallecido, y de esta manera se expresa en las actas capitulares de 1737 :

"Vista una petición del Raz[ione]ro D. Martín García Arevalillo, Alvacea, y heredero del Raz[ione]ro M[aest]ro de Capilla D. Miguel de Ambiela, en que dice, que aviendo recaído en él por herencia los Papeles de Música de d[ic]ho $\mathrm{M}$ [aest]ro, los ofrece, y da al Cab[ild]o por sí y en n[omb] re de d[ic]ho M[aest]ro, para que las obras de este logren el lucim[ien]to que se merecen en el Choro de esta S[an] ta Ig[lesi]a: acordaron, se le estime a d[ic]ho Raz[ione]ro Arevalillo esta oferta, y que se haga Imbentario de éstas, y de las demás que [h]avía, el qual firme el Raz[ione]ro M[aest] ro de Capilla D. Jaime Casellas, y esté a su cargo, y tenga la llave, para que dé quenta de $\mathrm{d}[\mathrm{ic}]$ hos papeles s[iem]pre que se le pida: quedando el S[eñ]or D. Carlos de Rivera, M[aestr] escuela en el cuidado de que no se saque ninguno $\sin \mathrm{su}$ orden, o del S[eñ]or que presida"27.

25 Missa sobre la Letanía de Nuestra Señora, Missa sobre el motete que dize Santa Maria, Missa sobre Popule meus, Missa sobre Iste cognovit Justitiam, Missa sobre el Ave Maria, Missa sobre el Veni sponsa Christi, todas ellas de Ardanaz.

26 Los cuatro primeros versos del soneto dicen:

Entre fragosas breñas emboscado

Do no pasaba gente ni se oía,

Gerónimo con llanto enternecía

Las fieras de aquel yermo despoblado...

Es un soneto de Juan López de Úbeda publicado en LÓPEZ DE ÚBEDA (1582), que recoge LABRADOR (2007).

27 ACT, 63, 01.08.1737, ff.172r.-172v.
La consecución de la entrega de los papeles de música y la realización del inventario se ejecutaron sin mayores consecuencias, es decir, no hay más cita que ésta en las Actas referida al asunto. Este inventario está mucho mejor organizado que el anterior; siguen agrupándose las obras por bloques enunciados por géneros litúrgicos, pero esta vez se distinguen primeramente las composiciones de Ambiela y después las de "maestros diferentes". Estos dos apartados se repiten por cada una de las especies de música latina de acuerdo al siguiente orden: misas $(9 / 13)^{28}$, magnificats $(8 / 11)$, Beatus vir (7/6), vísperas $(1 / 0)$, Dixit Dominus (11/9), otros salmos de vísperas y de nona (9/13), Salves $(5 / 15)$, varias $(7 / 4)^{29}$, motetes $(17 / 6)$, salmos de completas (2/4), lamentaciones (4/6) y misereres (19/3). Al final, fuera del orden establecido previamente, se añaden un motete del Rosario, dos pares de vísperas y el Oficio de Nuestra Señora de los dolores, vísperas, missa y motete, en quadernos de pergamino metidos en su caja de madera, que se citaba ya en el inventario que procedía de Ardanaz.

En total se recogen 191 composiciones, de las cuales 111 son de Ambiela y 80 de otros maestros. Ya he referido anteriormente el decepcionante saldo de obras de Ambiela que nos ha quedado hoy en el archivo de Toledo, y de ahí la importancia de un documento como éste para descubrir la verdadera dimensión de un maestro tan representativo de la música eclesiástica española de la primera mitad del siglo XVIII. En la siguiente tabla podremos ver con detalle la capacidad creativa que Ambiela desplegó en la catedral de Toledo durante sus 23 años de magisterio, ampliando los recursos musicales hasta los límites que le permitían su cargo y el tipo de repertorio en el que tenía que emplearlos:

Tabla I.- Inventario de obras latinas de Miguel de Ambiela, $1737^{30}$

MISAS (10)

\begin{tabular}{|l|}
\hline $\begin{array}{l}\text { Misa de instrumentos sobre el himno Quem terra, en } 4 \text { coros } \\
\text { de voces y uno de instrumentos (2 copias) }\end{array}$ \\
\hline Misa a 8 sobre el Saeculorum de $4^{\circ}$ tono \\
\hline Misa a 10 \\
\hline Misa a 11 con violines \\
\hline Misa de difuntos \\
\hline Misa a 8 Ecce Sacerdos magnus \\
\hline Misa a 8 con 2 violines y oboe sobre el himno de Apóstoles \\
\hline Misa a 8 con 2 violines a Nuestra Señora de la Concepción \\
\hline Misa a 8 con 2 violines y oboe sobre el himno Celestis Urbs \\
\hline
\end{tabular}

28 En el paréntesis, separados por una barra, primero el número de obras de Ambiela pertenecientes a ese género litúrgico y después el de otros maestros.

29 Invitatorio e Himno de Navidad, Invitatorio de Nuestra Senora, Monstra te esse, salmo de maitines, Victimae Paschali laudes.

30 Los géneros se citan en el mismo orden del documento. 
MAGNIFICATS (8)

\begin{tabular}{|l|}
\hline Magnificat a 8 con 2 violines y clarín \\
\hline Magnificat a 8 con violines $y$ oboe \\
\hline Magnificat a 8 \\
\hline Magnificat a 8 \\
\hline Magnificat a 12 \\
\hline Magnificat a 11 \\
\hline Magnificat a 12 \\
\hline Magnificat a 8 con 2 violines \\
\hline
\end{tabular}

\section{BEATUS VIR (7)}

Beatus vir a 8 con 2 violines

Beatus vir a 8 con 2 violines $y$ oboes

Beatus vir a 12

Beatus vir a 8 con 2 violines y oboe

Beatus vir a 17 con 2 violines y oboes

Beatus vir a 8

Beatus vir a 8

\section{VÍSPERAS (3)}

Unas Vísperas enteras a 4 con duplicado, con 2 violines y oboe con los salmos Dixit Dominus, Beatus vir, Laetatus sum y Magnificat al estilo italiano.

Dos pares de Vísperas a 8. Dixit Dominus, Beatus vir, Laudate Dominum, Laetatus sum, Lauda Jerusalem y Magnificat en cuadernos de papel

\section{DIXIT DOMINUS (11)}

Dixit Dominus a 8 con 2 violines y oboe

Dixit Dominus a 12

Dixit Dominus a 8

Dixit Dominus a 8 con 2 violines y oboe

Dixit Dominus a 8 con 2 violines

Dixit Dominus a 15 con 2 violines

Dixit Dominus a 8 con 2 violines y oboes

Dixit Dominus a 11

Dixit Dominus a 12

Dixit Dominus a 11 con chirimías

Dixit Dominus a 8

\section{LAETATUS SUM (3)}

Letatus sum a 8 con 2 violines y oboe

Letatus sum a 8

Letatus sum a 12 con bajones

\section{CREDIDI (1)}

Credidi a 8 con 2 violines y oboe
LAUDATE DOMINUM (3)

Laudate Dominum a 8 con 2 violines

Laudate Dominum a 8

Laudate Dominum a 8

SALMOS DE NONA (2)

Salmo de Nona $1^{\circ}$

Salmo de Nona $3^{\circ}$

SALVE REGINA (5)

Salve Regina a 12

Salve Regina a 10

Salve Regina a 12 con 2 violines y oboe

Salve Regina a 8

Salve Regina a 8

DIFERENTES (6)

(INVITATORIOS, HIMNOS Y SALMOS DE MAITINES)

Invitatorio e himno de Navidad

Himno de Navidad a 6

Invitatorio de Nuestra Señora de la Paz.

Himno de Completas

Monstra te esse a solo con 2 violines

Primer salmo de maitines a 6

SECUENCIAS (1)

Victimae Paschali laudes a 8

\section{MOTETES (18)}

Motete a la Ascensión a 8

Motete de un mártir a 8 con 2 violines

Motete a 8 de Confesor non Pontifice

Motete a 8 Veni Sponsa Christi

Motete a 8 San Francisco de Asis

Motete a 12 a San Francisco de Asis

Motete a 8 a la Dedicación

Motete a 8 a la Asunción

Motete a 12 con bajoncillos a Santiago

Motete a 8 Veni electa mea

Motete a 8 Ecce Sacerdos magnus

Motete a 8 a los santos Justo y Pastor

Motete a la Santísima Trinidad

Motete a 12 Iste Sanctus

Motete a San Francisco de Asis

Motete a Santa Teresa de Jesús

Motete a 8 Veni Sancte Spiritus

Motete del Rosario, Padre Nuestro

ANUARIO MUSICAL, N. ${ }^{\circ} 73$, enero-diciembre 2018, 135-152 ISSN: 0211-3538 doi:10.3989/anuariomusical.2018.73.09 
SALMOS DE COMPLETAS (3)

\begin{tabular}{|l|}
\hline Qui habitat a 10 \\
\hline Cum invocarem a 12 \\
\hline Nunc dimittis a 10 \\
\hline
\end{tabular}

LAMENTACIONES $\left(10^{31}\right)$

\begin{tabular}{|l|}
\hline $\begin{array}{l}\text { Lamentaciones de Miércoles, Jueves y Viernes Santo a } 8 \text { con } \\
\text { violines en } 12 \text { cuadernos }\end{array}$ \\
\hline Lamentación a 12 \\
\hline
\end{tabular}

\begin{tabular}{|c|}
\hline $\begin{array}{l}\text { MISERERES (19) } \\
\end{array}$ \\
\hline Miserere a 6 \\
\hline Miserere con instrumentos \\
\hline Miserere a 12 con 2 violines \\
\hline Miserere con bajoncillos \\
\hline Miserere \\
\hline Miserere a 8 con 2 violines \\
\hline Miserere \\
\hline Miserere a 4 con duplicado \\
\hline Miserere a 8 con 2 violines \\
\hline Miserere \\
\hline Miserere con bajoncillos \\
\hline Miserere a 16 con 2 violines y oboes \\
\hline Miserere con instrumentos \\
\hline Miserere con 2 violines \\
\hline Miserere a 20 con 2 violines y oboes \\
\hline Miserere a 16 con 2 violines y oboes \\
\hline Miserere a 8 \\
\hline Miserere a 8 duplicado con 2 violines y oboes \\
\hline Miserere con 2 violines \\
\hline OFICIOS (1) \\
\hline $\begin{array}{l}\text { Oficio de Nuestra Señora de los Dolores: Víspera, Misa y } \\
\text { motete en cuadernos de pergamino metidos en su caja de } \\
\text { madera }\end{array}$ \\
\hline
\end{tabular}

TOTAL $=111$

De los muchos detalles que nos ofrece esta larga lista de obras, hay algunos que llaman la atención de manera especial. Por ejemplo, de las diez misas que se mencionan -todas ellas perdidasse puede deducir una evolución del género en la trayectoria del mismo compositor. La Missa sobre el himno Quem terra, seguramente compuesta antes de la llegada de Ambiela a Toledo, pues ya se mencionaba en el inventario de Ardanaz, está concebida para 17 voces distribuidas en cuatro coros, de los que uno de ellos se especifica que era para instrumentos; esta distribución policoral y el empleo

31 Suponiendo que los doce cuadernos contengan las tres lamentaciones de cada día. de los instrumentos de viento equiparado a las voces eran prácticas habituales en el siglo XVII. De otro lado, el progresivo predominio de las misas, magnificats, salmos o motetes a 8 voces con violines y oboes que se muestra en buena parte de la lista, manifiestan la tendencia por la elección de este formato, que será el más característico de la música eclesiástica española del siglo XVIII, en el que la configuración espacial del coro (esto es, el lugar en el que se produce la música) con dos órganos enfrentados en tribunas opuestas, invita a la partición en dos del conjunto de la capilla.

De manera singular, Ambiela manifiesta también su interés por otras maneras de hacer música diferentes a las que le son más familiares; así, encontramos en el manuscrito anotadas unas "Vísperas enteras a 4 con duplicado, con violines y oboe (...) al estilo italiano". No ajenos a las novedades que los rodeaban, la creciente incorporación de músicos transalpinos a las capillas españolas, así como el gusto italianizante de la cercana corte, hacían que los maestros que estaban al frente de la música en las catedrales, se mostrasen cautamente interesados en probar diferentes estilos, y la música a 4 voces con instrumentos concertantes se asociaría a Italia como una práctica de referencia.

Otra sección llamativa en el índice de obras de Ambiela es la de los 19 misereres que se mencionan; al no haberse conservado ninguno, y tratarse del maestro de capilla que antecedió a Jaime Casellas, del que se conservan 24 misereres, no sabemos si éste siguió la estela del maestro aragonés en cuanto a la confección del género o si introdujo una nueva manera de concebirlo basada en las características de los suyos que conocemos. Éstas se ajustan a una idea en la que hay una sucesión de variadas secciones contrastadas, determinadas por las estrofas que impone el texto, en las que se desgranan múltiples combinaciones vocales e instrumentales (a menudo con destacadas partes solistas), y enmarcadas por grandes efectos policorales al principio y al final.

La intencionalidad de buscar estos efectos policorales en algunos misereres de Ambiela (se cita uno para 20 voces con violines y oboes) es evidente, pero ignoramos cuál era el desarrollo interno en el transcurso de toda la obra.

La otra gran aportación que nos ofrece la lectura del inventario de 1737 es la de proporcionarnos una extensa relación de compositores españoles tanto contemporáneos de Ambiela como de generaciones atrás, lo que de por sí constituye una antología de la música religiosa de los cien años anteriores a la fecha del manuscrito. Se mencionan un total de 27 nombres de músicos activos en diferentes épocas, que podríamos clasificar de la siguiente manera:

a) Activos en la $1^{\text {a }}$ mitad del siglo XVII: Juan Bautista Comes, Vicente García, Carlos Patiño y Urbán de Vargas.

b) Activos en la $2^{\mathrm{a}}$ mitad del siglo XVII y $1^{\mathrm{a}}$ década del XVIII: Sebastián Alfonso, Pedro de Ardanaz, Diego Cáseda, Juan de Ciurana, Sebastián Durón, Cristóbal Galán, José Hinojosa, Miguel J. Marqués, Tomás Micieces, Juan Pérez Roldán y Matías Veana.

c) Activos en la $1^{\text {a }}$ mitad del siglo XVIII: Fermín de Arizmendi, Joaquín Martínez de la Roca, Juan Manuel Sánchez Organero, Gregorio Portero, Blas de San José, Matías Solana, José de Torres, Francisco Valls y Enrique Manuel Villaverde. 
Por otra parte, podemos también relacionar los nombres que se citan por áreas geográficas de las que, en algunos casos coinciden con los lugares por los que pasó el maestro Ambiela en su trayectoria profesional. De este modo, hallamos algunos músicos que trabajaron en algún momento en el área aragonesa como Alfonso, Cáseda, Marqués, Pérez Roldán, Urbán de Vargas o Martínez de la Roca ${ }^{32}$; este último, además de coincidir con Ambiela en Toledo desde su admisión como organista en 1722, estuvo en El Pilar de Zaragoza a la vez que aquel en una etapa anterior ${ }^{33}$.
Uno de los principales méritos que podría tener cualquiera de los nombres que aparece en la lista sería el de estar relacionado con alguna de las capillas de la corte (Capilla Real, monasterio de las Descalzas Reales, Real convento de La Encarnación). Así, podemos encontrar varios de estos ilustres maestros que cumplieron toda o alguna etapa profesional en uno de estos lugares; en ciertos casos, incluso hubo quienes pasaron de un convento a otro.

Tabla II.- Maestros de las capillas reales que se citan en el inventario de 1737:

\begin{tabular}{|c|c|c|}
\hline Capilla Real & Descalzas Reales & La Encarnación \\
\hline & J. Pérez Roldán (1655-1662) & Carlos Patiño (1628-1634) \\
Juan B. Comes (1618-1632) & Tomás Micieces (1662-1667) & Vicente García (1634-1645) \\
Carlos Patiño (1634-1675) & Cristóbal Galán (1667-1680) & J. Pérez Roldán (1648-1655) \\
Sebastián Durón (1691-1706) & Matías Veana (1683-1689) & Matíana (1680-1683) \\
Cristóbal Galán (1680-1684) & Bonet de Paredes (1691-1706) & J. Bonet de Paredes (1687-1691) \\
José de Torres (1686-1738) & Miguel de Ambiela (1707-1710) & Pedro Rodrigo (1744-1750) \\
\hline
\end{tabular}

En un tercer grupo podemos integrar a aquellos músicos que, de alguna manera, estuvieron vinculados con la catedral de Toledo. Como maestros de capilla anteriores a Ambiela, figuran en el inventario los nombres de todos los que ocuparon el puesto desde mediados del siglo XVII ${ }^{34}$, a excepción de Juan de Padilla, de quien habíamos visto en el inventario de 1713 que el cabildo aceptaba una donación de veinticuatro obras procedentes de Córdoba, y de las que ahora no se hace mención. No obstante, la presencia de composiciones de estos maestros toledanos anteriores a Ardanaz es bastante parca.

En el inventario también aparecen citados dos organistas que trabajaron en Toledo al servicio de Ambiela en sucesivas etapas: Matías Solana, hasta 1722, y Joaquín Martínez de la Roca que ocupó el mismo puesto tras la muerte de aquél. Martínez alcanzó más prestigio y consideración que Solana y ejerció labores de maestro de capilla de manera no oficial cuando Ambiela enfermó en sus últimos años de vida, llegando incluso a encargarse de la composición de la música en ocasiones muy especiales, como las que se produjeron en 1732 cuando se festejaron las solemnidades para la inauguración del Transparente de la catedral que diseñara Narciso Tomé en la cabecera del templo.

Por último, observamos también la relación de los maestros que obtuvieron su formación en Toledo partiendo de su condición de seises, una vez perdida la voz, siendo reconducidos entonces hacia una educación musical que les posibilitase

32 Todos ellos pasaron en distintas épocas por El Pilar de Zaragoza, salvo Sebastián Alfonso, que estuvo en la Seo.

33 Miguel de Ambiela fue maestro de capilla en El Pilar de Zaragoza entre los años 1700 y 1707, mientras que Joaquín Martínez de la Roca fue organista allí mismo entre 1695 y 1715.

34 La sucesión de maestros de capilla de Toledo representados en el manuscrito parte de Vicente García (1645-1650), y continúa con Tomás Micieces (1650-1662), -después iría Juan de Padilla (1662-1673)-, Pedro de Ardanaz (1674-1706) y Juan Bonet de Paredes (1707-1710). optar por un magisterio de capilla en alguna institución española que lo requiriera. En esta categoría se nombran cinco casos, tres de ellos discípulos de Pedro de Ardanaz, pues salen destinados a otra catedral al poco de llegar Ambiela. Se trata de Gregorio Portero (a Granada, 1713), Fermín de Arizmendi (a Ávila, 1714) y Juan Manuel de la Puente (a Jaén, 1716). Solo un pupilo de Ambiela logra un magisterio: Enrique Manuel Villaverde, admitido como seise en 1714, obtendrá el magisterio de Oviedo diez años después; Juan Manuel Sánchez Organero, que entró como seise en Toledo a la par que Villaverde, hubiera obtenido otro puesto similar si no hubiera sido por su prematura muerte en 1732, después de haberlo intentado sin éxito en Zamora un año antes. Las obras que se citan de todos estos que fueran seises, que una vez más no se han conservado, se reflejan en número de una o dos por cada caso, y muy bien podrían haber sido fruto de su período de aprendizaje.

Y hasta aquí mis comentarios. A quien haya interesado lo expuesto, descubrirá más detalles en la lectura de los propios documentos, cuya transcripción encontrará en los apéndices del final de este artículo. He considerado oportuno además, aportar una tabla en la que figuren los datos esenciales de los maestros que aparecen en ambos inventarios, añadiendo los nombres y el número de las obras que de ellos se mencionan ${ }^{35}$.

35 Sería prolijo enumerar una a una las fuentes documentales o bibliográficas de donde procede cada dato de los que se citan en esta tabla. Todos los datos referidos a Toledo proceden de las diversas fuentes que he venido consultando en los últimos años en el archivo de la catedral (Actas Capitulares, Libros de Obra y Fábrica, Libros de Sucesión de Prebendas, etc.) y muchos de los cuales se recogen en mi estudio sobre la música de la catedral en el siglo XVIII (MARTÍNEZ GIL, 2003) o en la tesis doctoral de Natalia MEDINA sobre el siglo XVII (2016). Por otro lado, las referencias a otros maestros no relacionados directamente 
APÉNDICE 1.- Compositores que se citan en el documento de obras latinas que fueron de Miguel de Ambiela y de Pedro de Ardanaz ${ }^{36}$

\begin{tabular}{|c|c|c|c|c|c|}
\hline & $\begin{array}{c}\text { Lugares en los que estuvo } \\
\text { activo }\end{array}$ & \begin{tabular}{|c|}
$\begin{array}{c}\text { Obras citadas en el inventario } \\
\text { de Ambiela }\end{array}$ \\
\end{tabular} & Total & $\begin{array}{c}\text { Obras citadas en el inventario de Ardanaz } \\
\text { + Obras de Padilla }\end{array}$ & Total \\
\hline $\begin{array}{c}\text { ALFONSO, } \\
\text { Sebastián }(* 1616 ; \\
\dagger 1692)\end{array}$ & $\begin{array}{c}\text { Albarracín (1637), Huesca } \\
\text { (1641), Cuenca (1653), La } \\
\text { Seo de Zaragoza (1656-1687) }\end{array}$ & $\begin{array}{c}\text { Magnificat a } 8(*) \\
\text { Dixit Dominus a } 10 \\
\text { Lauda Jerusalem a } 8\end{array}$ & 3 & & \\
\hline $\begin{array}{c}\text { AMBIELA, } \\
\text { Miguel de }(* 1666 ; \\
\dagger 1733)\end{array}$ & $\begin{array}{c}\text { Daroca (1681), Lleida } \\
\text { (1686-1690), Jaca (1698), } \\
\text { El Pilar de Zaragoza (1700) } \\
\text { Descalzas Reales (1707), } \\
\text { Toledo (1710-1733) }\end{array}$ & $\begin{array}{l}\text { Véase tabla con las obras de } \\
\text { Ambiela en su inventario }\end{array}$ & 111 & $\begin{array}{c}\text { Dixit Dominus a } 8\left(i^{*} ?\right) \\
\text { Beatus vir a } 8\left(i^{*} ?\right) \\
\text { Laetatus sum a } 8\left(i^{*} ?\right)(\text { los tres salmos con } \\
\text { violines }) \\
\text { Magnificat a } 8 \text { con violines } \\
\text { Missa a } 8 \text { con violines y oboes } \\
\text { Oficio de Ntra } \text { Sr }^{a} \text { de los Dolores }(*) \\
\text { Visperas y Missa } \\
\text { Missa a diez y siete con instrumentos sobre } \\
\text { el himno Quem terra } \\
\end{array}$ & 9 \\
\hline $\begin{array}{c}\text { ARDANAZ, Pedro } \\
(* 1638 ; \dagger 1706)\end{array}$ & $\begin{array}{c}\text { Seise en Toledo, } \\
\text { Pamplona (1658), } \\
\text { Toledo (1674-1706) }\end{array}$ & $\begin{array}{c}\text { Laudate Dominum a } 8 \\
\text { Salve a } 10 \\
\text { Salve a } 9 \\
\text { Salve a } 9 \\
\text { Salve a } 12 \\
\text { Salve a } 6 \\
\text { Miserere a } 12\end{array}$ & 7 & Véase el inventario de Ardanaz & 196 \\
\hline $\begin{array}{l}\text { ARIZMENDI, } \\
\text { Fermín de } \\
(* 1691 c ; \uparrow 1733)\end{array}$ & $\begin{array}{l}\text { Seise en Toledo, } \\
\text { Ávila (1714-1733) }\end{array}$ & $\begin{array}{l}\text { Motete a } 8 \text { Similabo eo } \\
\text { Motete a } 8 \text { a San Pedro Apóstol }\end{array}$ & 2 & & \\
\hline $\begin{array}{c}\text { BONET DE } \\
\text { PAREDES, Juan } \\
(* 1647 \mathrm{c} ; \dagger 1710)\end{array}$ & $\begin{array}{c}\text { Berlanga de Duero (1680), } \\
\text { Ávila (1682), Segovia (1684), } \\
\text { La Encarnación (1687), } \\
\text { Descalzas Reales (1691), } \\
\text { Toledo (1706-1710) }\end{array}$ & $\begin{array}{l}\text { Memento Domine a } 8(*) \\
\text { Domine probasti a } 10\end{array}$ & 2 & & \\
\hline $\begin{array}{l}\text { CÁSEDA, Diego } \\
(* 1638 \mathrm{c} ; \dagger 1694)\end{array}$ & $\begin{array}{l}\text { Tudela (1658), Logroño, } \\
\text { Zamora (1668), El Pilar de } \\
\text { Zaragoza (1673-1694) }\end{array}$ & $\begin{array}{c}\text { Misa a } 12 \\
\text { Magnificat a } 8(*)\end{array}$ & 2 & & \\
\hline $\begin{array}{l}\text { CIURANA, } \\
\text { Juan de }{ }^{37} \\
(?)\end{array}$ & Área valenciana & \begin{tabular}{|c|} 
Magnificat a 8 \\
Dixit Dominus a 8 \\
Credidi a 8 \\
Monstra te esse Matrem a solo y a 9 \\
Monstra te esse Matrem a 5
\end{tabular} & 5 & & \\
\hline
\end{tabular}

con Toledo han sido consultadas de inicio en el Diccionario de la Música Española e Hispanoamericana (2002). Comoquiera que desde la publicación de estos volúmenes hasta la actualidad se han realizado numerosos estudios monográficos que afectan a alguno de los nombres que figuran en los inventarios, en la medida de lo posible he intentado recoger estos nuevos datos para actualizar la información que se pretende ofrecer en esta tabla. Algunos de estos estudios más recientes aparecen reseñados en la bibliografía final de este artículo.

36 Con (*) las obras que aún se conservan en el Archivo de la catedral de Toledo.

37 El apellido Ciurana aparece citado en cinco ocasiones en el inventario de Ambiela de 1737, cuatro de ellas como "Ciurana", sin más, y una como "M[aest]ro Ciurana". No he encontrado información alguna referida a este maestro; el hecho de haberle situado en el área valenciana se debe a la existencia de otros músicos bastante posteriores reconocidos con este apellido: Joaquín (activo en la $2^{\mathrm{a}}$ mitad del s.XVIII), Manuel (activo en la $1^{a}$ mitad del s.XIX) y Tomás (*Peñíscola, 1762; †Xátiva, 1829) [PERSONAT (2011)]. Por otra parte, la atribución de Juan al maestro citado en el inventario de Ambiela, cuando en ningún momento se menciona este nombre, es porque en un inventario posterior, fechado en 1775, se le incluye como autor de tres obras que aún perviven de entonces: "Del Músico d[o]n Juan de Siurana / Un dixit d[omi]nus, y un Credidi todo a 8 / El Himno Monstra te ese Matrem a 5" (Yndice de las obras latinas que [h]oy se hallan en la Papelera de esta santa Primada Yglesia, de varios Autores, hasta el M[aest]ro Casellas inclusive. Cuio arreglo se hizo este presente Año de 1775). No se le vuelve a mencionar en ninguno de los inventarios conservados de fechas posteriores. 


\begin{tabular}{|c|c|c|c|c|c|}
\hline $\begin{array}{c}\text { COMES, Juan } \\
\text { Bautista }(* 1582 \mathrm{c} ; \\
\dagger 1643)\end{array}$ & $\begin{array}{c}\text { Colegiata de Gandía (1606), } \\
\text { Corpus Christi de Valencia } \\
\text { (1608), catedral de Valencia } \\
\text { (1613), Capilla Real (1618), } \\
\text { Valencia (1632) }\end{array}$ & Magnificat a 8 & 1 & & \\
\hline $\begin{array}{l}\text { DURÓN, } \\
\text { Sebastián } \\
(* 1660 ; \dagger 1716)\end{array}$ & \begin{tabular}{|} 
La Seo de Zaragoza (1674), \\
Sevilla (1680), El Burgo de \\
Osma (1685), Palencia (1686), \\
Capilla Real (1691-1706)
\end{tabular} & \begin{tabular}{|} 
Misa a 7 con 2 violines y clarín $(*)^{38}$ \\
Lamentación con 2 violines \\
Lamentación a 8
\end{tabular} & 3 & & \\
\hline $\begin{array}{c}\text { GALÁN, Cristóbal } \\
(* 1615 \mathrm{c} ; \dagger 1684)\end{array}$ & \begin{tabular}{|c|} 
Teruel (1646), Cagliari (1654), \\
Morella (1656-1659), Madrid \\
(mtro."de los manestriles \\
negrillos del Buen Retiro", \\
esclavos del rey, 1657-1664), \\
Segovia (1664), Descalzas \\
Reales (1667-1675-[1680?]), \\
Capilla Real (1680-1684) \\
\end{tabular} & $\begin{array}{c}\text { Magnificat a } 4 \text { de difuntos } \\
\text { Beatus vir a } 8 \\
\text { Dixit Dominus a } 8\end{array}$ & 3 & & \\
\hline $\begin{array}{l}\text { GARCÍA, Vicente } \\
(* 1593 ; \dagger 1650)\end{array}$ & $\begin{array}{c}\text { Orihuela (1610), Valencia } \\
(1618), \text { Cuenca (1632), La } \\
\text { Encarnación (1634), Toledo } \\
\text { (1645-1650) }\end{array}$ & $\begin{array}{c}\text { Lauda Jerusalem a } 8 \\
\text { Lauda Jerusalem a } 11 \\
\text { Salve a } 4 \\
\text { Cum invocarem a } 8 \\
\text { Qui habitat a } 8\end{array}$ & 5 & $\begin{array}{c}\text { Motete a } \mathrm{Ntr}^{a} \mathrm{Sr}^{a} \text { Sancta et Immaculata a } 8 \\
\text { Magnificat a } 8 \\
\text { Missa a } 12 \text { sobre Entre fragrosas breñas }\end{array}$ & 3 \\
\hline $\begin{array}{c}\text { HINOJOSA, José } \\
(\dagger 1673)\end{array}$ & \begin{tabular}{|c|} 
Calatayud (hasta 1658), \\
Teruel, Colegio del Patriarca \\
de Valencia (1662-1673)
\end{tabular} & $\begin{array}{c}\text { Ave Regina } \\
\text { Motete a } 10 \text { a la Ascensión } \\
\text { Cum invocarem a } 13 \\
\end{array}$ & 3 & & \\
\hline $\begin{array}{l}\text { MARQUÉS, } \\
\text { Miguel Juan } \\
\text { (activo entre } \\
\text { fl.1641-1661) }\end{array}$ & $\begin{array}{c}\text { Daroca (1641), El Pilar de } \\
\text { Zaragoza (1653) }\end{array}$ & $\begin{array}{c}\text { Beatus vir a } 10 \\
\text { Dixit Dominus a } 9\end{array}$ & 2 & & \\
\hline $\begin{array}{l}\text { MARTÍNEZ } \\
\text { DE LA ROCA, } \\
\text { Joaquín } \\
(* 1676 \mathrm{c} ; \dagger 1747)\end{array}$ & $\begin{array}{l}\text { El Pilar de Zaragoza (1695, } \\
\text { Palencia (1715), Toledo } \\
(1722-1747)\end{array}$ & \begin{tabular}{|c|} 
Misa a 8 con 2 violines y \\
bajoncillos \\
Misa a 8 sobre los himnos del Corpus \\
Misa a 8 de difuntos con violines \\
Misa a 8 con violines y oboes \\
Salve a 10 con violón \\
Miserere a 5 coros
\end{tabular} & 6 & & \\
\hline $\begin{array}{l}\text { MICIECES, } \\
\text { Tomás } \\
(* 1624 ; \dagger 1667)\end{array}$ & $\begin{array}{c}\text { León }(1646), \text { Toledo }(1650) \\
\text { Descalzas Reales }(1662- \\
1667)\end{array}$ & $\begin{array}{l}\text { Dixit Dominus a } 8 \\
\text { Dixit Dominus a } 12\end{array}$ & 2 & $\begin{array}{c}\text { Dixit Dominus a } 13 \\
\text { Magnificat a } 8(*) \\
\text { Magnificat a } 10(*) \\
\text { Monstra te esse a } 3 \text { con dos bajones } \\
\text { Lección de difuntos a } 12 \\
\text { Motete Sepulto Domino a } 12(*)\end{array}$ & 6 \\
\hline $\begin{array}{c}\text { ORGANERO, } \\
\text { Juan Manuel } \\
\text { Sánchez } \\
(* 1701 ; \dagger 1732) \\
\end{array}$ & $\begin{array}{c}\text { Seise en Toledo, un año } \\
\text { antes de su muerte oposita } \\
\text { al magisterio de Zamora sin } \\
\text { éxito }\end{array}$ & \begin{tabular}{|c|} 
Lamentación a solo con dos violines \\
Lamentación a 4 \\
Lamentación a 4 \\
Lamentación a solo con dos violines
\end{tabular} & 4 & & \\
\hline $\begin{array}{c}\text { PADILLA, Juan } \\
\text { de }(* 1605 ; \dagger 1673)\end{array}$ & $\begin{array}{c}\text { Coria, La Seo de Zaragoza, } \\
\text { Zamora (1661-1662), Toledo } \\
(1662-1673)\end{array}$ & & & $\begin{array}{c}\text { Diez cuadernos de psalmos de vísperas a } 7 \\
\text { Dos Missas a } 7 \\
\text { (Véase el añadido final al inventario de Ardanaz) }\end{array}$ & $\begin{array}{l}3+24 \\
=27\end{array}$ \\
\hline
\end{tabular}

38 Solo se conserva la portada del clarín y fragmentos de los violines. 


\begin{tabular}{|c|c|c|c|c|c|}
\hline $\begin{array}{l}\text { PATIÑO, Carlos } \\
(* 1600 ; \dagger 1675)\end{array}$ & $\begin{array}{c}\text { Mozo de coro en Sevilla, m } \\
\text { de canto del Sagrario en la } \\
\text { catedral de Sevilla (1623), } \\
\text { La Encarnación (1628), } \\
\text { Capilla Real (1634) }\end{array}$ & $\begin{array}{c}\text { Misa a } 8 \\
\text { Magnificat a } 8 \\
\text { Beatus vir a } 8 \\
\text { Dixit Dominus a } 8 \\
\text { Lauda Jerusalem a } 8 \\
\text { Credidi a } 8(*) \\
\text { Laetatus sum a } 8(*) \\
\end{array}$ & 7 & Motete a $\operatorname{Ntr}^{a} \mathrm{Sr}^{a}$ a 8 Maria Mater Dei & 1 \\
\hline $\begin{array}{c}\text { PORTERO, } \\
\text { Gregorio } \\
(* 1692 ; \dagger 1755) \\
\end{array}$ & $\begin{array}{c}\text { Seise en Toledo, } \\
\text { Granada (1713-1755) }\end{array}$ & Invitatorio & 1 & & \\
\hline $\begin{array}{c}\text { PÉREZ } \\
\text { ROLDÁN, Juan } \\
(* 1604 ; \dagger 1672 \mathrm{c})\end{array}$ & $\begin{array}{l}\text { Berlanga de Duero (1636), } \\
\text { tenor y m de seises en } \\
\text { Toledo (1636), Calahorra } \\
\text { (1639), Málaga (1642), } \\
\text { La Encarnación (1648), } \\
\text { Descalzas Reales (1655), } \\
\text { Segovia (1664), León (1670), } \\
\text { El Pilar de Zaragoza (1671) } \\
\end{array}$ & $\begin{array}{c}\text { Misa a } 12 \\
\text { Misa a } 12 \\
\text { Magnificat a } 12\end{array}$ & 3 & Missa de Batalla a 12 & 1 \\
\hline $\begin{array}{c}\text { PUENTE, Juan } \\
\text { Manuel de la } \\
(* 1692 ;+1753) \\
\end{array}$ & $\begin{array}{c}\text { Seise en Toledo, Jaén (1716- } \\
1753)\end{array}$ & $\begin{array}{c}\text { Magnificat a } 8 \\
\text { Dixit Dominus a } 8\end{array}$ & 2 & & \\
\hline $\begin{array}{c}\text { RODRIGO, Pedro } \\
(\dagger 1750)\end{array}$ & $\begin{array}{c}\text { Oviedo (1722), Santiago } \\
\text { (1723),La Encarnación (1744) }\end{array}$ & $\begin{array}{c}\text { Laetatus sum a } 8 \text { con } 2 \text { violines } \\
\text { y oboe }\end{array}$ & 1 & & \\
\hline $\begin{array}{c}\text { ROGIER, } \\
\text { Philippe }(* 1561 \mathrm{c} ; \\
+1596) \\
\end{array}$ & Capilla Real flamenca & & & $\begin{array}{l}\text { Regina coeli a } 8 \\
\text { Missa a } 12\end{array}$ & 2 \\
\hline 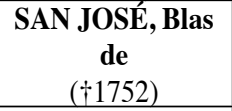 & Guadalupe & Regina coeli a 12 & 1 & & \\
\hline $\begin{array}{c}\text { SOLANA, Matías } \\
(\dagger 1722)\end{array}$ & $\begin{array}{c}\text { Organista en Toledo desde } \\
1689 \text { hasta } 1722 \\
\end{array}$ & Magnificat a 8 & 1 & Vísperas y Missa & 2 \\
\hline $\begin{array}{c}\text { TORRES, } \\
\text { José de } \\
(* 1670 \mathrm{c} ; \dagger 1738)\end{array}$ & $\begin{array}{c}\text { Capilla Real, organista desde } \\
1686 \text { y m}^{\circ} \text { de capilla desde } \\
1718\end{array}$ & $\begin{array}{c}\text { Misa a } 8 \text { con dos violines y clarín } \\
\text { Beatus vir a } 8 \text { con dos violines y } \\
\text { oboe }(*) \\
\text { Letatus sum a } 8 \text { con violines y oboe }\end{array}$ & 3 & & \\
\hline $\begin{array}{c}\text { VALLS, Francisco } \\
(* 1671 \mathrm{c} ; \dagger 1747)\end{array}$ & Barcelona (1696-1726) & \begin{tabular}{|c|} 
Misa a 8 \\
Magnificat a 10 con dos violines $(*)$ \\
Salve a 7 \\
\end{tabular} & 3 & & \\
\hline $\begin{array}{l}\text { VARGAS, Urbán } \\
\text { de } \\
(* 1606 ; \dagger 1656)\end{array}$ & $\begin{array}{c}\text { Huesca (1629), Pamplona } \\
\text { (1629), Calatayud (1637), } \\
\text { El Pilar de Zaragoza (1645), } \\
\text { Burgos (1651), El Pilar } \\
(1653), \text { Valencia (1653) }\end{array}$ & Salve a 8 & 1 & & \\
\hline $\begin{array}{c}\text { VEANA, } \\
\text { Matías Juan } \\
(* 1656 ; \dagger 1708)\end{array}$ & $\begin{array}{c}\text { Parroquia de San Juan del } \\
\text { Mercado de Valencia (1677), } \\
\text { La Encarnación (1680), } \\
\text { Descalzas Reales (1683) }\end{array}$ & $\begin{array}{c}\text { Misa a } 8 \\
\text { Magnificat a } 8 \\
\text { Beatus vir a } 8, \\
\text { Dixit Dominus a } 8 \\
\text { Credidi a } 8(*) \\
\text { Motete a la Transfiguración }(*)\end{array}$ & 6 & & \\
\hline $\begin{array}{c}\text { VICENTE, } \\
\text { Gerónimo }(\dagger 1648) \\
\end{array}$ & $\begin{array}{l}\text { Vitoria, Tafalla, Calahorra, } \\
\text { Santiago, Barbastro } \\
\end{array}$ & & & Missa a 8 & 1 \\
\hline $\begin{array}{c}\text { VILLAVERDE, } \\
\text { Enrique Manuel } \\
(* 1702 ; \dagger 1774)\end{array}$ & $\begin{array}{l}\text { Seise en Toledo, Oviedo } \\
\text { (1724-1774) }\end{array}$ & Miserere a 8 & 1 & & \\
\hline $\begin{array}{c}\text { Nombres y obras } \\
\text { citados }=\end{array}$ & Nombres citados $=\mathbf{2 8}$ & $\mathrm{N}^{\mathrm{o}}$ de obras $=$ & 191 & $\begin{array}{r}\text { Nombres citados }=\mathbf{1 0} \\
\mathrm{N}^{0} \text { de obras }=\end{array}$ & 248 \\
\hline
\end{tabular}


APÉNDICE 2. Inventario de obras latinas que fueron de Pedro de Ardanaz, 1713.

Documento I.- Imbentario $^{39}$ de las obras de Lattin de $D^{n}$ Pedro de Ardanaz, Razionero y Maestro de Capilla que fue de la muy Santta Yglesia desta Ziudad Primada de las Españas, las q[ua]les obras dejó a esta S[an]ta Ig[lesi]a como consta del acto cap[itula]r de 3 de s[eptiemb]re de 1706, diose a la hered[er]a una gratificaz[io]n de 20 dob[lone]s por acto cap[itula]r de 6 de Ab[ri]l de 713.

$Y$ de otras obras del M[aest]ro Padilla, q[ue] paraban en Cordova y los restituieron a esta S[an]ta Ig[lesi]a Prim[a] da como se dice en el acto cap[itula]r de $1^{\circ}$ de jul[i]o de 706. Cab[ild]o 21 de s[eptiemb]re de 712

$\mathrm{q}[\mathrm{ue}]$ todas esta obras se pongan en el cajón de las tribunillas con las demás q[ue] allí ay y de todas se haga entrega al M[aest]ro de Capilla actual para q[ue] las guarde y de quenta de ellas siempre q[ue] se le pida por el imbent[ari]o q[ue] se guarde en la Obra.

Primeram[en]te nueve Misas, en la forma siguiente

Una Missa de a veinte vozes con chirimías de quinto tono

Otra a doze sobre la Letania de N[uest]ra S[eñor]a de octavo

tono

Otra a doze sobre un Motete que dize Santa Maria de primer tono

Otra a doze de Vatalla, del M[aestr]o Roldan de quinto tono

Otra a onze sobre Populle meus de tercer tono

Otra a ocho, sobre Iste cognovit Justitiam de tercer tono

Otra a ocho sobre el Ave Maria de sexto tono

Otra a ocho sobre el Veni sponsa Christi de octavo tono

Otra a ocho del M[aestr]o Geronimo Vicente de octavo tono Motetes

Un Motete, a doze vozes, de San Ildefonso, q[ue] empieza Gaudete de segundillo

Otro a doze para la festividad de la Dedicazion de la yglessia, q[ue] empieza Domus mea de segundillo

Otro para todos los Santos a doze, q[ue] empieza Angeli Archangeli, de octavo tono

Otro del Dulzisimo Nombre de Maria a doze que empieza, Refugium est, de quinto tono

Otro a doze de N[uestr]a S[eñor]a que empieza Quam gloriosa, de segundillo

Otro a doze de S[an] Juan Baptista, q[ue] empieza Precursor Domini, de primer tono

Otro a doze de la Ascension, q[ue] empieza Aszendens Christus in altum, de octavo tono

Otro a doze de S[an]to Thomas de Aquino que empieza, O Doctor Angelice, de segundillo

Otro a doze de la Concepcion de N[uestr]a S[eñor]a que empieza, Conceptio tua, de tercer tono

Otro a doze de la Asumpcion de N[uestr]a S[eñor]a que empieza Quae est ista, de octavo tono_

Otro a doze Para N[uestr]a S[eñor]a de el Sagrario, que empieza Sacraris Virgini, de primer tono nuscrito.

39 Los subrayados y las letras iniciales mayúsculas son del ma-
Otro a onze de San Pedro, q[ue] empieza Tu es Pastor ovium, de séptimo tono

Otro a onze de Santiago, q[ue] empieza Iste est qui ante Alios, se sexto tono

Otro a 10 de la S[antí]s[i]ma Trinidad, que empieza Duo Seraphin, de octavo tono

Otro a diez Para S[an]to Domingo de Guzman que empieza Te Dominum celebrant, de segundo tono

Otro a ocho para S[an] Eugenio q[ue] empieza Iste cognovit, de tercer tono

Otro a ocho de N[uestr]a S[eñor]a q[ue] empieza Sancta Maria sucurre miseris, de primer tono

Otro a ocho de N[uestr]a S[eñor]a del Maestro Patiño que empieza. Maria Mater Dei, de segundo tono

Otro a ocho Para San Julian, q[ue] empieza Argentum aut Aurum, de octavo tono

Otro a ocho Para S[an] Ignacio de Loyola q[ue] empieza Sint lumbi bestri, de octavo tono

Otro a ocho Para Virgines q[ue] empieza, Veni sponsa Christi, de octavo tono

Otro a ocho Para N[uestr]a S[eñor]a del Rosario, q[ue] dize Ave Maria, de sexto tono

Otro a ocho a N[uestr]a S[eñor]a del M[aestr]o Vicente Garzia q[ue] empieza Sancta et Inmaculata de quinto tono

Otro a N[uestr]a S[eñor]a de los dolores, a siete, q[ue] empieza $\mathrm{O}$ bos omnes, de segundo tono

Otro a ocho q[ue] empieza Elegit eos Dominus, de segundillo

Otro al S[antí]s[i]mo S[acramen]to a nueve, q[ue] empieza Beati omnes, de octavo tono

Otro al S[antí]s[i]mo S[acramen]to a ocho, q[ue] empieza Jubilate Deo, de primer tono

Otro al S[antí]s[i]mo S[acramen]to a seis q[ue] empieza O quam suabis est, de segundillo

Dos Sequenzias

Una de Pasqua de Resurreczion a doze que empieza Victime Paschali, de primer tono

Otra de Pasqua de Spiritu Santo a doze q[ue] empieza Veni Santi Spiritus, de primer tono

Nona

Dos psalmos para nona, Dia De la Ascension

Uno Mirabilia testimonia tua a catorze con chirimías de octavo tono

Otro Principes persecuti sunt me gratis a diez, de séptimo tono Visperas

Diez Psalmos de Dixit Dominus q[ue] son los siguientes.

Uno a diez y seis vozes, de segundo tono

Otro, a quinze de octavo tono

Otro, a doze de primer tono

Otro a treze, del M[aestr]o Micieces de $7^{\circ}$ tono

Otro a doze con chirimías, de octavo tono

Otro a onze de séptimo tono

Otro a ocho de quinto tono

Otro a ocho de primer tono

Otro a ocho de quinto tono

Otro a ocho de primer tono

Ocho Psalmos de Beatus vir, en la forma siguiente

Uno a diez y siete vozes de octavo tono

Otro a doze se segundo tono 
Otro a doze, de octavo tono

Otro a catorze, de sexto tono

Otro a diez de octavo tono

Otro a ocho de séptimo tono

Otro a ocho, de segundo tono

Otro a ocho, de segundo tono

Zinco psalmos de Letatus sum, q[ue] son los siguientes.

Uno a treze vozes, de segundo tono

Otro a doze, de primer tono

Otro a diez, de octavo tono

Otro a ocho, de sexto tono

Otro a ocho, de sexto tono

Quatro psalmos de Lauda Jerusalem, en la forma siguiente.

Uno a doze vozes, de segundo tono

Otro a doze, del mismo tono

Otro a ocho, de séptimo tono

Otro a ocho del mismo tono

Zinco psalmos de Laudate Dominum omnes gentes, q[ue] se siguen a la vuelta

Uno de a doze vozes, de séptimo tono

Otro a diez, de octavo tono

Otro a ocho, de tercer tono

Otro a ocho, de el mismo tono

Otro a quatro, de segundo tono

Dos psalmos de Credidi, q[ue] son los siguientes

Uno a doze de octavo tono

Otro a ocho de segundo tono

Un psalmo a doze Inegistu [sic] Isrrael de Egipto

Otro psalmo a ocho Memento Domine David, de séptimo tono

Otro psalmo a ocho, Domine probasti me de octavo tono

Doze Magnificas, q[ue] son los siguientes

Una a diez y seis con chirimías de $5^{\circ}$ tono

Otra a catorze de sexto tono

Otra a doze, de Primer tono

Otra a doze, de sexto tono

Otra a diez del M[aestr]o Micieces, de $7^{\circ}$ tono

Otra a ocho del M[aestr]o Micieces de $4^{\circ}$ tono

Otra a ocho de octavo tono

Otra a ocho de quarto tono

Otra a ocho del mismo tono

Otra a ocho de octavo tono

Otra a ocho de tercer tono

Otra a ocho del M[aestr]o Vicente Garzia de octavo tono

Doze himnos de Visperas a 4 q[ue] son los sig[uien]tes

Uno de S[an] Joseph. Octavo tono

Otro de S[an]ta Theresa del mismo tono

Otro de S[an] Ermenegildo de $4^{\circ}$ tono

Otro de S[an] Vicente Ferrer de $1^{\circ}$ tono

Otro del Dulcissimo N[ombr]e de Jesus de $1^{\circ}$ tono

Otro de la Corona de espinas de $8^{\circ}$ tono

Otro de Santiago de quinto tono

Otro De los Dolores de primer tono

Mas otro de los dolores del mismo tono

Otro de N[uestr]a S[eñor]a que dize Ave Maris Stela a veinte vozes de primer tono

Otro de N[uestr]a S[eñor]a Ave Maris stella a 12

Mas un Monstra te ese Matrem, a quatro con instrumentos
Otro a quatro con instrumentos

Otro a tres, con instrumentos

Otro a tres con dos vajones del Maestro Micieces

Completas

Nuebe psalmos de Completas q[ue] son los siguientes.

Cum imbocarent a 13 de segundo tono

Otro a 12 de octavo tono

Otro a 8 de segundo tono

Qui hábitat a 12 de segundo tono

Otro a 10 del mismo tono

Otro a 8 de segundo tono

Nunc dimitis a 10 de tercer tono

Otro a 9 de segundo tono

Otro a 8 de tercer tono

Un himno para las Completas a 8. Que dize, te lucis ante terminum de segundillo

Mas Ave Regina coelorum, a 12 para después de las Completas de N[uestr]a S[eñor]a de la Encarnazion de $1^{\circ}$ tono

Mas Regina coeli letare a 12 de sexto tono para las de el Sabado S[an]to

Otra Regina coeli a 8 del mismo tono del M[aestr]o Phelipe Rogier

Siete Letanias de N[uestr]a S[eñor] en la forma sig[uien]te

Una a 12 vozes de quinto tono

Otra a 12 de octavo tono

Otra a 10 de segundillo

Otra a 8 de tercer tono

Otra a 8 de segundo tono

Otra a 7 del mismo tono

Otra a 4 de primer tono

Mas otra Letania de el S[antí]s[i]mo Sacramento de sexto tono

Memoria de los Papeles de Latin del Maestro $\underline{\text { Don }} \underline{\underline{\text { Pedro }}} \underline{\underline{\text { de }}}$ $\underline{\text { Ardanaz }}$

Dixit Dominus ----- 08

Beatus vir ----- $\quad 05$

Laudate dominum ----- 03

Letatus sum ----- 06

Lauda Jerusalem ----- $\quad 04$

Magnifica Anima mea ----- 11

Motetes a diferentes santos ----- $\quad 24$

Salve Regina ---- 06

Letanias a N[uestr]a S[eñor]a -----

Regina coeli ----- 02

Ave Regina coelorum ----- 01

Psalmos de Completas

Cum ymbocarem -----

03

Qui abitat -----

Himno te lucis ante -----.

Nun dimitis ----- 02

Psalmos de Nona

Mirabilia -----

01

Principes -----

01

Oficio de Difuntos

Regem cuy omnia vibunt ----- $\quad 02$

Parce mihi domine ---- 01

Motetes de difuntos ----- $\quad 02$

Tedet animam meam ----- $\quad 01$ 
Domine probasti me ----- 01

Lamentationes ----- 05

Motetes de quaresma ----- 17

Himmo de S:" Joseph (tachado) -----

Missa a doze voces ----- 01

Missa a doze voces sobre la Letania ----- $\quad 01$

Missa a veinte con chirimías ----- 01

Missa a diez voces ----- 01

Missa a doze voces de Requiem

en quadernos de pergaminos ----- $\quad 01$

Missa a ocho voces sobre el motete

Histe cognovit ----- 01

Nuebe quadernos en papel de psalmos.

Dixit dominus. y Beatus vir a 8 voces --- $\quad 01$

Domine Dominus noster ----- 01

Bisperas y Missa de D. ${ }^{\mathrm{n}}$ Mathias Solana organista

[En el margen izquierdo se indica que las siguientes obras son de Ambiela]

Dixit dominus a ocho con violines.

Beatus vir. a ocho con violines.

Letatus sum. a ocho con violines.

Magnificat. a ocho con violines.

Missa. a ocho con violines y oboes

el oficio de N[uestr]a S[eñor]a de los Dolores

Visperas y Missa del Maestro D. ${ }^{n}$ Miguel de Ambiela en quadernos de pergamino en su caja

Missa adiez y siete con ynstrumentos. sobre el himno Quem terra. echa por D. ${ }^{\mathrm{n}}$ Miguel de Ambiela.

[En el margen izquierdo] Padilla

Diez quadernos de Psalmos en papel de vísperas a siete voces del Maestro D. ${ }^{\mathrm{n}}$ Juan de Padilla

Missa a doze voces entre fragrosas Breñas del M[aestr]o Vicente Garcia

Dos Missas en quadernos de pergamino a siete voces del Maestro D. ${ }^{\mathrm{n}}$ Juan de Padilla

Missa a doze voces del Maestro Phelipe Rogier en quadernos de pergamino

Catorze salves, En la forma siguiente

Una a 14 vozes primer tono

Otra a treze

Otra a doze

Otra a doze

Otra a doze

Otra a onze

Otra a onze

Otra a diez

Otra a diez

Otra a nueve

Otra a nueve

Otra a ocho

Otra a siete

Otra a seis

Dos psalmos para Maytines de N[uestr]a S[eñor]a q[ue] son los sig[uien]tes

Uno, Empieza Domine Dominus noster a 12 de séptimo tono

El otro es la misma letra, a seis de segundillo

Ofizio de Difuntos

Ymbitatorio, regem cui omnia a doze de sexto tono
Una lección, q[ue] empieza Parce Mihi Dom[in]e a doze, de primer tono

Otra a 12 . Parce mihi, de $6^{\circ}$ tono

Otra a 8 . Parce mihi, de $2^{\circ}$ tono

Otra lección q[ue] empieza Tedet Anima mea, a 12 del M[aestr]o Micieces de octavo tono

Una Misa de Requiem a doze de sexto tono con Motete, que esta enquadernada y forrada en pergamino tiene 14 libretes Otro Motete suelto a 12 Para Reyes difuntos, q[ue] empieza Spiritus meus, de segundo tono

\section{Quaresma}

Veinte y un motetes de Algunas Dominicas, y ferias de quaresma a quatro

Semana Santa

Siete Lamentaciones en esta forma

Miercoles

Una lección prima fer [ia] $5^{a}$ in Cena Domini a quinze, q[ue] Empieza Incipit lamentatio, de segundo tono

Otra, lectio $1^{a}$ fer[ia] $5^{a}$ in Cena Domini a doze de primer tono

Juebes S[an]to

Otra leccio $1^{a}$ fer[ia] sexta in Parasceve que dize De lamentatione a 12 de séptimo tono

Otra leccio $1^{a}$ fer[ia] sexta in Parasceve a ocho de séptimo tono

Otra leccio $1^{a}$ fer[ia] sexta in Parasceve a 4 de séptimo tono Otra leccio $3^{a}$ fer[ia] sexta in Parasceve q[ue] empieza Aleph, A duo, de $7^{\circ}$ tono

Viernes S[an]to

Un Motete a doze, de el M[aestr]o Micieces que empieza Sepulto Domino, que se canta acabado el sermón de las tres oras

Una lamentación para este dia $1^{a}$ a 12 que empieza, de lamentatione, de séptimo tono

Otra leccio $2^{a}$ q[ue] empieza Aleph, sola de sexto tono

Mas quinze Misereres de los grandes Para Miercoles y Juebes Santo

Mas otros seis Misereres Para el Viernes Santo en esta forma

Uno a Doze

Otro a onze

Otros dos a ocho

Otro a siete

Yotro a seis

[Rúbrica al final de esta lista]

Papeles del M[aestr]o D. Juan de Padilla M[aes]tro de Cap[ill]a que fue de esta S[ant]a Iglesia.

P[rimeramen]te Una Missa de dos vajos a 7 con pergam[in]

$o$

Una Missa, y oficio de difuntos a 8

Unas Visperas a 7. en quadernos

Un Dixit D[omi]n[u]s, y beatus vir a 8. en quadernos

Un Dixit D[ominu]s a 7 en pap[ele]s y otro a 8.

Dos lauda hierusalem a 8

Unos Dixit D[ominu]s Beatus vir y Magnif[ica]t a 12

Un letatus sum a 12.

Un Magnificat a 8.

Una letania al S[antísi]mo a 8

Un te Deum laudamus a 8 
El himno a S[an] hermenegildo a 4

Dos motets a la Asump[cio]n a 11 y a 8

Un motete de S[an] Miguel a 8

Unos Psalmos de Com[leta]s

Una Prosa de Resurreccion a 10

APÉNDICE 3. Inventario de papeles de música latinos que fueron de Miguel de Ambiela, 1737.

Documento II.- Nota de los papeles de Musica latinos, que fueron del Raz[ione]ro M[aest]ro de Capilla de esta S[an]ta Igl[esi]a, $D^{n}$. Miguel de Ambiela (que D[io]s aya y presenta al Ill[ustrisi]mo Card[dena]l para q[ue] se sirva de ellos (si su S[eño]ria Ill[lustrisi]ma gusta) el D[octo]r $D^{n}$. Martin de Arevalillo Raz[ione]ro de esta S[an]ta Igl[esi]a.

Ambiela ${ }^{40}$

Primeram[en]te una Missa de Ynstrum[en]tos sobre el Hymno Quem terra, en quatro coros de voces, y uno de Ynstrum[en]tos

Una Missa a 8 sobre el Seculorum de $4^{\circ}$ tono

Una Missa a 10

Una Missa a 11 con violines

Una Missa de difuntos

Una Missa a 8 ecce Sacerdos magnus

Una Missa a 8 con 2 violines, y obue, sobre el Hymno de Apostoles

Una Missa a 12 = Dedicada a Maria Santisima

Una Missa a 8 con 2 violines $=a N^{a} S .^{r a}$ de la Conzepcion

Una Missa a 8 con 2 violines, y obue, sobre el Himno Celestis Urbs.

Mas otra copia de la Missa sobre el himno Quem terra

De M[aest]ros diferentes

Una Missa a 8 del M[aest]ro Patiño

Una Missa a 7 con 2 violines y clarín del M[aest]ro Durón.

Una Missa a 12 del M[aest]ro Roldan

Una Missa a 8 con 2 violines y clarín del M[aest]ro Torres.

a San Pedro Arbues [Esto último, apuntado por olvido en el margen izquierdo]

Una Missa a 8 con 2 violines y vajoncillos de D. ${ }^{n}$ Joachin Martinez.

Una Missa a 8 del M[aest]ro Valls

Una Missa a 8 del M[aest]ro Patiño

Una Missa a 8 del M[aest]ro Veana

Una Missa a 12 del M[aest]ro Casseda

Una Missa a 12 del M[aest]ro Roldan

$\underline{\text { Una Missa a } 8 \text { sobre los Hymnos de Corpus de D. }}{ }^{n} \underline{\text { Joachin }}$ Martinez

Una Missa a 8 de Difuntos. con violi[ne]s de D. $\underline{\underline{n} \text { Joachin }}$ Martinez

$\underline{23}$ [En el margen izquierdo, al final de la lista de misas] [A la vuelta de esta misma hoja, se completan las misas con una más de Joaquín Martínez]

40 Los subrayados que vayan apareciendo son originales. El nombre de Ambiela, al igual que el de las siguientes distinciones de autores de las obras, se escriben en el margen izquierdo del folio.
Missas - 24 - Una Missa a 8. con violi[ne]s y oboes de $D^{n}$ Joachin Martinez

Ambiela

Una Magnificat a 8 con 2 violines, y clarín

Otra Magnificat a 8 con violines, $y$ obue

Otra Magnificat a 8

Otra Magnificat a 8

Otra Magnificat a 12

Otra Magnificat a 11

Otra Magnificat a 12

Otra Magnificat a 8 con 2 violines

De M[aest]ros diferentes

Una Magnificat a 10 con 2 violines, del M[aest]ro Valls

Otra Magnificat a 8 del M[aest]ro Ciurana.

Otra Magnificat a 8 del M[aest]ro Veana.

Otra Magnificat a 8 del M[aest]ro Alphonso

Otra Magnificat a 12 del M[aest]ro Roldan.

Otra Magnificat a 8 del M[aest]ro Comes

Otra Magnificat a 8 del M[aest]ro Casseda.

Otra Magnificat a 8 del M[aest]ro de Solana

Otra Magnificat a 8 del M[aest]ro Patiño

Otra Magnificat a 8 del M[aest]ro Puente

Otra Magnificat a 4, de Difuntos, del M[aest]ro Galán

$\underline{19}$ [En el margen izquierdo, al final de la lista de magnificats] Ambiela

Un Beatus vir a 8 con 2 violines

Otro Beatus vir a 8 con 2 violines, y oboes

Otro Beatus vir a 12

Otro Beatus vir a 8 con 2 violines, y oboe

Otro Beatus vir a 17 con 2 violines, y oboes

Otro Beatus vir a 8

Otro Beatus vir a 8

De M[aest]ros diferentes

Un Beatus vir a 10 del M[aest]ro Marques

Otro Beatus vir a 10 con ynstrum[en]tos del M[aest]ro Ardanaz.

Otro Beatus vir a 8 del M[aest]ro Patiño

Otro Beatus vir a 8 del M[aest]ro Veana

Otro Beatus vir a 8 del M[aest]ro Galan

Otro Beatus vir a 8 con 2 violines, y oboe, del M[aest]ro Torres

$\underline{13}$ [En el margen izquierdo, al final de la lista de Beatus vir] Ambiela

Unas Visperas enteras a 4 con duplicado, con 2 violines y oboe con los Psalmos, dixit Dominus, Beatus vir, Laetatus sum: y Magnificat. al estilo ytaliano

Un dixit Dominus a 8 con 2 violines, y oboe

Otro dixit Dominus a 12

Otro dixit Dominus a 8

Otro dixit Dominus a 8 con 2 violines, y oboes

Otro dixit Dominus a 8 con 2 violines

Otro Dixit Dominus a 15 con 2 violines

Otro dixit Dominus a 8 con 2 violines, y oboes

Otro dixit Dominus a 11

Otro dixit Dominus a 12

Otro dixit Dominus a 11 con chirimías

Otro dixit Dominus a 8

De M[aest]ros diferentes

Otro dixit Dominus a 8 del M[aest]ro Miciesses

Otro dixit Dominus a 12 
Otro dixit Dominus a 9 del M[aest]ro Marques

Otro dixit Dominus a 10 del M[aest]ro Alphonso

Otro dixit Dominus a 8 de Ciurana.

Otro dixit Dominus a 8 del M[aest]ro Patiño

Otro dixit Dominus a 8 del M[aest]ro la Puente

Otro dixit Dominus a 8 del M[aest]ro Galan

Otro dixit Dominus a 8 del M[aest]ro Veana

Son dixit Dominus, con los Psalmos Beatus vir, Laetatus sum, y Magnificat de las Visperas enteras 24

$\underline{24}$ [En el margen izquierdo, al final de la lista de Dixit Dominus] $\underline{\text { Ambiela }}$

Laetatus sum a 8 con 2 violines, y oboe

Credidi a 8 con 2 violines, $y$ oboe

Laudate Dominum omnes gentes a 8 con 2 violines

Otro a 8

Otro a 8

Laetatus sum a 8

Otro a 12 con vajones [la terminación "es" está tachando

"cillos", es decir, se corrige vajoncillos por vajones]

$1^{o}$ y $3^{\circ}$ Psalmos de nona

De M[aest]ros diferentes

$2^{\circ}$ Psalmo de nona

Laetatus sum a 8 con violines, y oboe, del M[aest]ro Torres

Otro a 8 con 2 violines y oboe, del M[aest]ro Rodrigo

Credidi a 8 del M[aest]ro Veana

Lauda Jerusalem a 8, del M[aest]ro Patiño

Otro a 8 del M[aest]ro Garzia

Otro a 11

Otro a 8 del M[aest]ro Alphonso

Laudate Dominum a 8 del M[aest]ro Ardanaz

Credidi a 8 de del M[aest]ro Patiño

Otro a 8 de Ciurana

Laetatus sum a 8 del M[aest]ro Patiño

Memento a 8, y Domine probasti a 10, del M[aest]ro Paredes

Son Psalmos de Visperas, con los 3 de nona, 25

$\underline{25}$ [En el margen izquierdo, al final de la lista de salmos]

Ambiela

Salve Regina a 12

Otro a 10

Otra a 12 con 2 violines, y oboe

Otro a 8

Otro a 8

De M[aest]ros diferentes

Una salve a 10 con violon de D. ${ }^{n}$ Joachin Martinez

Otra a 7 del M[aest]ro Valls

Otra a 10 de Solana

Otra a 5

Otra a 10 del M[aest]ro Ardanaz.

Otra $a$ $9=$

Otra a 8 de Fr. Blas de $S^{n}$. Joseph

Otra a 9 del M[aest]ro Ardanaz.

Otra a 12 del M[aest]ro Ardanaz.

Otra a 4 del M[aest]ro Garzia

Otra a 6 del M[aest]ro Ardanaz.

Otra a 8 de Solana

Otra a 8 del M[aest]ro Bargas

Ave Regina coelorum del M[aest]ro Ynojosa

Regina coeli laetare a 12 de Fr. Blas de $S^{n}$. Joseph
20 [En el margen izquierdo, al final de la lista de las antífonas marianas]

Diferentes

Ynvitatorio y Hymno de Navidad del M[aest]ro Ambiela

Hymno de Nav[ida]d a 6 del M[aest]ro Ambiela

Ynvitatorio de N[uest]ra S[eño]ra de la Paz, del M[aest]ro

Ambiela

Otro del M[aest]ro Portero

Ynvitatorio de Nav[ida]d a 8

Hymno de Completas a duo del M[aest]ro Ambiela

Monstra te ese Matrem a solo, y a 9 de Ciurana

Monstra te ese Matrem a 5 de Ciurana

Monstra te ese Matrem a solo con 2 violines, del M[aest] ro Ambiela

Primer Psalmo de Maytines a 6

Son Ynvitatorios, Hymnos, y el Psalmo de Maytines 10

$\underline{10}$ [En el margen izquierdo, al final de la lista]

Ambiela

Victime Paschali laudes a 8

Motete a la Ascension a 8

Motete de un Martir a 8 con 2 violines

Motete a 8 de Confesor non Pontifice

Motete a 8 Veni sponsa Christi

Motete a S. ${ }^{\mathrm{n}}$ Fran[cis]co de Asis

Otro a 12 al mismo S[an]to

Motete de la Dedicazion a 8

Motete de la Asumpcion a 8

Motete a 12 con vajoncillos a Santiago

Motete a 8 Veni electa mea

Motete a 8 ecce sacerdos magnus

Motete a 8 a S. ${ }^{\mathrm{n}}$ Justo y Pastor

Motete a la S[antí]s[i]ma Trinidad

Motete a 12 Iste Sanctus

Motete a S. ${ }^{\mathrm{n}}$ Fran[cis]co de Asis

Motete a S[an]ta Theresa de Jesus

Motete a 8 Veni Sancte Spiritus

De M[aest]ros diferentes

Motete a la Transfiguraz[io]n del M[aest]ro Veana

Motete a 8: Similabo eum, del M[aest]ro Arizmendi

Mote [sic] a 8 a N[uest]ra S[eño]ra del M[aest]ro Veana

Motete a 8 a S. ${ }^{n}$ Pedro Apostol, de Arizmendi

Motete a 10 a la Ascension, del M[aest]ro Ynojosa

Motete a 12 de difuntos, del M[aest]ro Roldan

$\underline{\mathbf{2 4}}$ [En el margen izquierdo, al final de la lista de motetes]

Diferentes

Cum invocarem a 8 del M[aest]ro Garzia

Qui habitat a 8 del M[aest]ro Garzia

Cum invocarem a 13 del M[aest]ro Ynojosa

Qui hábitat a 10 del M[aest]ro Ambiela

Cum invocarem a 12

Nunc dimitis a 10 del M[aest]ro Ambiela

Psalmos de Completas todos seis

6 [En el margen izquierdo, al final de esta última lista]

Lamentaz[ion]es de diferentes.

Lamentaziones de Miércoles, Juebes, y Viernes S[an]to a 8

con violines en 12 Quadernos del M[aest]ro Ambiela

Otra Lamentaz[i]on a 12 del M[aest]ro Ambiela

Otra Lamentac[i]on a solo con 2 violines, de Organero 
Otra a 4

Otra a 4

Otra a solo con 2 violines

Otra con 2 violines del M[aest]ro Durón

Otra a 8

$\underline{10}$ [En el margen izquierdo, al final de las lamentaciones] Ambiela

Miserere a 6

Mi[s]erere con ynstrum[en]tos

Miserere a 12 con 2 violines

Miserere con vajoncillos

Otro Miserere. Miserere a 8 con 2 violines. Otro Miserere

Miserere a 4 con duplicado

Papeles de Miserere sueltos

Verso de Miserere con 2 violines, y oboes

Miserere a 8 con 2 violines

Otro Miserere

Miserere con vajonzillos

Miserere a 16 con 2 violines, y oboes

Miserere con ynstrum[en]tos

Miserere con 2 violines

Miserere a 20 con 2 violines, y oboes

Miserere a 16 con 2 violines, y oboes

Miserere a 8

Miserere a 8 duplicado con 2 violines, y oboes

Miserere con 2 violines

De diferentes

Miserere a 8 del M[aest]ro Villaverde

Miserere a 12 del M[aest]ro Ardanaz.

Miserere a 5 choros de D.n Joachin Martinez

$\underline{\mathbf{2 4}}$ [En el margen izquierdo, al final de la lista de todos los misereres]

Un motete del Rosario, Padre n[uest]ro del Maestro Ambiela [Hay una rúbrica al final de la hoja]

M[aest]ro Ambiela

Dos pares de Vísperas a 8. Dixit dominus, Beatus vir, Laudate Dominum, Letatus sum, Lauda Jerusalem, y Magnificat. En quadernos de papel

M[aest]ro Ambiela

Un oficio de Nuestra Señora de los Dolores. Visperas, Missa, y motete, en quadernos de pergamino metidos en su caja de madera

Me he dado, y me doy desde luego por entregado de todos los papeles contenidos en este quaderno que contiene quatro ojas, y me obligo a dar q[uen]ta de ellos a los Ill[ustrísi]mos S[eño]res Dean y Cab[ild]o y Obra de esta S[an]ta Igl[esi] a, y en caso de mi fallecim[ien]to encargo a mis Alvaceas, que den q[uen]ta de ellos como deuda que $\mathrm{p}[\mathrm{o}] \mathrm{r}$ este recibo y obligación contraigo, y lo firme en Toledo a veintey seis de se[ptien]vre de mil setez[ient]os y treinta y siete.

Nota de los papeles de música q[ue] fueron de D. Miguel de Ambiela Racion[e]ro y M[aest]ro de Capilla. 1737. $\underline{22}$

\section{BIBLIOGRAFÍA}

Actas Capitulares de la Catedral de Toledo (ACT).

Álvarez Escudero, Carmen M., El Maestro aragonés Miguel de Ambiela (1666-1733). Su contribución al Barroco musical, Oviedo, Servicio de Publicaciones de la Universidad de Oviedo, 1982.

Calahorra Martínez, Pedro, Historia de la música en Aragón (siglos I-XVII). Zaragoza, Librería General, 1977.

Casares Rodicio, Emilio; Fernández de la Cuesta, Ismael; López-Calo, José, Diccionario de la Música Española e Hispanoamericana, Madrid, SGAE, 2002.

Ezquerro Esteban, Antonio, Villancicos policorales aragoneses del siglo XVII, Barcelona, CSIC, 2000.

Guillén, María Cristina, y Ruiz de Elvira, Isabel: Catálogo de Villancicos y Oratorios en la Biblioteca Nacional. Siglos XVIII-XIX, Madrid, Ministerio de Cultura, Dirección General del Libro y Bibliotecas, 1990.

Jiménez Criado, Francisco, Tomás Micieces, maestro de capilla de la catedral de Toledo (1650-1662). Vida, obra y estilo musical en la España del siglo XVII. Trabajo de Máster en Patrimonio Histórico. Toledo, Facultad de Humanidades, Universidad de Castilla-La Mancha, 2013.

Labrador Herraiz, José L., Cancionero Sevillano B 2495 de la Hispanic Society of America, Sevilla, Universidad de Sevilla, 2007.

López-Calo, José, Catálogo del Archivo de Música de la Catedral de Ávila, Santiago de Compostela, Sociedad Española de Musicología, 1978.

López de Úbeda, Juan, Vergel de flores divinas, Alcalá de Henares, Juan Íñiguez de Lequerica, 1582.

Martínez Gil, Carlos, La Capilla de música de la catedral de Toledo (1700-1764). Evolución de un concepto sonoro, Toledo, Servicio de Publicaciones de la Junta de Comunidades de Castilla-La Mancha, 2003.

Medina Hernández, Natalia, La vida musical en la catedral de Toledo durante el siglo XVII, Madrid, Universidad Autónoma (Biblos-e Archivo), 2016.

Personat Remolar, Alfredo, Metodología analítica comparada de procedimientos compositivos y estilísticos en el período ilustrado hispánico: música de tecla inédita en Villareal (Castellón). Manuel Ciurana, Juan Moreno y Polo, José Ferrer et alii. Tesis Doctoral, Valencia, Universidad Politécnica de Valencia, 2011.

Rodilla León, Francisco, "La música en el Real Monasterio de Santa María de Guadalupe durante la 'Edad de Plata' (Siglo XVIII)", en Nassarre, 28 (Zaragoza, 2012), pp. 67-115.

Ruiz de Elvira, Isabel: Catálogo de Villancicos de la Biblioteca Nacional. Siglo XVII, Madrid, Ministerio de Cultura, Dirección General del Libro y Bibliotecas, 1992.

Sánchez Mombiedro, Rafael, La capilla musical del Real Colegio-Seminario del Corpus Christi en la segunda mitad del siglo XVII: biografía crítica, legado musical y magisterio de José Hinojosa (fl. 1662-†1673). Tesis Doctoral, Valencia, Universidad Politécnica de Valencia, 2016. 